\title{
RADIATION-INDUCED SOLUTE SEGREGATION IN METALLIC ALLOYS
}

\author{
Alan J. Ardell \\ Department of Materials Science and Engineering \\ Henry Samueli School of Engineering and Applied Science \\ University of California, Los Angeles \\ Los Angeles, CA 90095-1595, USA \\ Corresponding Author email address: aardell@ucla.edu \\ Pascal Bellon \\ College of Engineering \\ Department of Materials Science and Engineering \\ University of Illinois at Urbana-Champaign \\ Urbana, IL 61801
}

\begin{abstract}
The subject of radiation-induced solute segregation (RIS) in metallic alloys is reviewed. RIS manifests itself in several different ways, including diffusion to point-defect sinks (dislocations, grain boundaries, voids, etc.), which can induce precipitation in undersaturated alloys, as well as self-organization of solute clusters and precipitation in defect-free material. Diffusion in dilute and concentrated alloys is highlighted, as are theories of RIS that include new ideas on diffusion of complexes involving coupling between fluxes of point defects and of solute atoms. Many important experimental observations are presented, including up-to-date findings using atom-probe tomography, with special emphasis on solute segregation in austenitic and ferritic steels. Results from computational modeling and theory are also presented and discussed in light of experimental findings. Examples illustrating the factors affecting RIS are shown and some important outstanding issues that impact the current understanding of RIS are described and discussed.
\end{abstract}

Keywords: Radiation damage; solute segregation; diffusion; clustering; rate-theory kinetics; computational modeling; austenitic and ferritic stainless steels 


\section{Introduction}

The irradiation of crystalline solids by energetic neutrons, ions or electrons subjects them to several kinds of radiation damage. Radiation damage begins with the production of atomic displacement damage, generally from collision cascades of various sizes that depend on the energy and mass of the bombarding species. The collisions produce vacancies and interstitial atoms in equal numbers, so-called Frenkel Pairs. Depending on the temperature of the materials the vacancy concentrations can far exceed their equilibrium values; the concentrations of interstitials are always far in excess of their equilibrium values, which are exceptionally small at all temperatures. A large fraction of the excess point defects recombine, eliminating the local radiation damage. In fact, it is fair to state that if recombination were perfect and all the vacancies and interstitials were eliminated by mutual annihilation, there would be no radiation damage at all except for transmutations that occur during neutron irradiation. But interstitials interact much more strongly than vacancies with both themselves and with pre-existing internal sinks for point defects, such as dislocations and grain boundaries, because of the large strain fields associated with the occupation of interstitial sites. Furthermore, interstitials are generally much more mobile than vacancies, so they have much shorter lifetimes. The interstitials that survive recombination interact with pre-existing sinks in a biased manner and leave behind the less mobile vacancies. Though vacancies are far less mobile than interstitials, they are nevertheless mobile enough to interact with each other, one serious consequence of which is the phenomenon of void swelling.

Interstitials and vacancies usually interact quite differently with the atomic species in an alloy. The interactions between atoms and point defects can be either attractive or repulsive, depending on factors such as size differences, electronic structure, etc. Moreover, the diffusive fluxes of interstitials or vacancies towards point-defect sinks often produce concomitant preferential fluxes of atoms. Even in the absence of strong binding energies between the point defects and atoms it is possible for atom fluxes to be induced by point-defect fluxes, leading to radiation-induced redistribution of solute and solvent atoms. The longer-range radiation-induced solute redistribution (RISR) at point-defect sinks is generally referred to as radiation-induced segregation (RIS). When or if the concentration of solute atoms at a sink exceeds the limit of solubility in the alloy a new phase can nucleate and grow, leading to radiation-induced precipitation (RIP). It is also possible in many cases for irradiation to produce solute 
redistribution on a much shorter length scale, resulting for example in the long-range order of a previously compositionally disordered alloy.

The current state of knowledge of RIS constitutes the focal point of this review article.

There have been several reviews of RIS over the past few decades [1-4] as well as review articles on radiation damage and reactor materials in general [5,6] and reactor steels in particular [7], and the interested reader is encouraged to peruse these articles to appreciate how our understanding of this topic has evolved over time. At this writing it is fair to state that advances in the processing of alloys to produce microstructural features at the nano-scale, characterizing them and modeling their radiation-damage resistance warrant a current assessment of the progress in our understanding of RISR. We present a historical perspective on RIS, discuss some of the most recent advances and observations, describe the different approaches to modeling RIS and discuss recent models that endeavor to predict behavior. The influence of experimental variables (e.g. radiation temperature, the addition of trace elements) and microstructural parameters (e.g. grain boundary character) will be highlighted, particularly in steels, which are candidate alloys for use in Generation IV nuclear reactors [6]. The effects of RISR on alloy behavior, particularly hightemperature mechanical properties and resistance to corrosion and stress-corrosion cracking, are normally quite deleterious. These issues are discussed in comprehensive review articles by Fukuya [5] and Zinkle and Was [6] and therefore will not be addressed in this paper. The advent of atom probe tomography (APT) has been highly impactful in characterizing defects at the nano-scale, and we present numerous examples of new observations made using this technique, the limitations and advantages of which have been reviewed recently by Marquis [8].

\section{Manifestations of Radiation-Induced Solute Redistribution}

Okamoto and Wiedersich [9] were the first to observe and identify both RIS and RIP, which was manifested by precipitation of the intermetallic compound $\mathrm{Ni}_{3} \mathrm{Si}$ (with the ordered $\mathrm{L}_{2} \mathrm{Cu}_{3} \mathrm{Au}$ crystal structure) at the surfaces of radiation-induced voids in an austenitic stainless steel. Okamoto and Wiedersich postulated that undersized Si atoms would preferentially populate the concentration of interstitial atoms. Since intersitials as well as vacancies diffuse towards void surfaces, which are very strong point-defect sinks, the precipitation of $\mathrm{Ni}_{3} \mathrm{Si}$ would occur once the solubility limit of this phase was exceeded. Shortly afterwards, Barbu and Ardell [10] observed the precipitation of $\mathrm{Ni}_{3} \mathrm{Si}$ in a Ni-4 at\% $\mathrm{Si}$ alloy irradiated by $\mathrm{Ni}^{+}$-ions at $500{ }^{\circ} \mathrm{C}$. 
Since the solubility of $\mathrm{Si}$ in Ni exceeds 10 at. \% at temperatures above $600{ }^{\circ} \mathrm{C}$ [11], it is evident that the $4 \% \mathrm{Si}$ alloy is highly undersaturated at $500{ }^{\circ} \mathrm{C}$. Moreover, the lattice constant of Ni-Si solid solutions decreases with increasing Si content [12], hence Si is clearly undersized in the binary alloy. One of the radiation-induced microstructures observed by Barbu and Ardell [10] is shown in Fig. 2.1; the segregation of Si to interstitial dislocation loops and precipitation on them is evident. The RIP of $\mathrm{Ni}_{3} \mathrm{Si}$ in undersaturated $\mathrm{Ni}-\mathrm{Si}$ alloys at grain boundaries, coherent twin boundaries and free surfaces was observed later by Janghorban and Ardell [13]; examples are shown in Fig. 2.2. The idea that RIS and RIP were due to a preferential interstitial population of undersized Si atoms was reinforced by the results of experiments by Rehn et al. [14] on binary $\mathrm{Ni}$ alloys containing 1 at. \% Si, $\mathrm{Al}, \mathrm{Ti}$ or Mo, the latter three of which are oversized. Using Auger spectroscopy, Rehn et al. found that Al, Ti and Mo segregated away from the free surface of their specimens; only Si segregated towards the free surface.
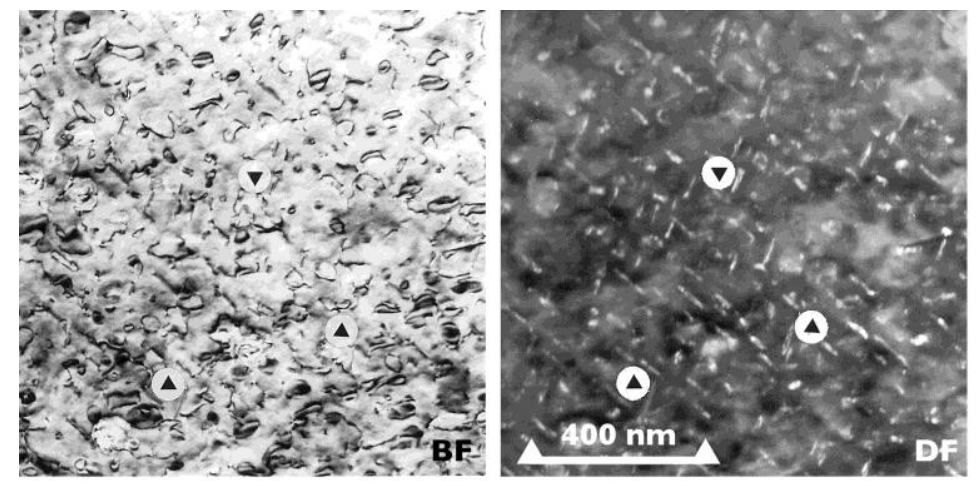

Figure 2.1. Bright field (BF) and dark field (DF) transmission electron micrographs of the same area (see arrowheads) in a Ni-6 at. \% Si alloy irradiated at $500{ }^{\circ} \mathrm{C}$ to a dose of $10 \mathrm{dpa}$ using $\mathrm{Ni}^{+}$ ions [10]. The precipitation of ordered $\mathrm{Ni}_{3} \mathrm{Si}$ is evident at the dislocation loops in the $\mathrm{DF}$ image, taken using a (100) superlattice reflection.

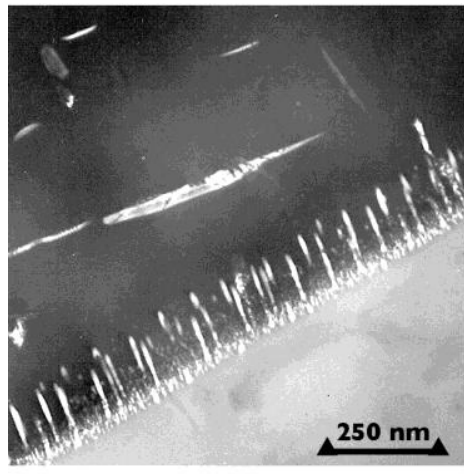

(a)

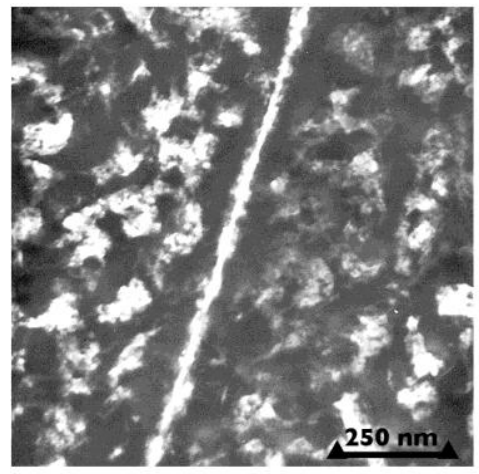

(b)

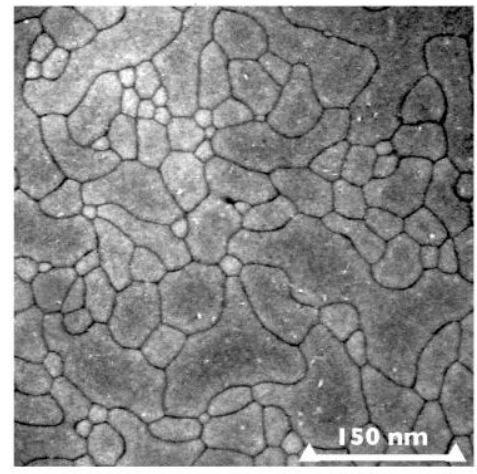

(c)

Figure 2.2. Dark-field images of 3 different manifestations of RIP in undersaturated Ni-Si alloys 
irradiated by $400 \mathrm{keV}$ protons: (a) discontinuous precipitation at a grain boundary, Ni-8 at.\% $\mathrm{Si}$, $0.42 \mathrm{dpa}, 450{ }^{\circ} \mathrm{C}$; (b) RIP at a coherent twin boundary, Ni-6 at.\% Si, 0.42 dpa $550{ }^{\circ} \mathrm{C}$; (c) a continuous surface film of $\mathrm{Ni}_{3} \mathrm{Si}$, showing the anti-phase domain boundary structure, Ni-6 at\% $\mathrm{Si}, 0.06 \mathrm{dpa}, 500{ }^{\circ} \mathrm{C}$. From Ardell and Janghorban [15].

Radiation-induced solute redistribution over shorter length scales is manifested in several different ways. We mention three examples here. The first is the introduction of long-range order in a disordered solid solution, exemplified by the discovery by Weaver and Ardell [16] of $\mathrm{Pd}_{8} \mathrm{~W}$ in an undersaturated alloy of $\mathrm{Pd}-18$ at.\% $\mathrm{W}$ irradiated by protons at $600{ }^{\circ} \mathrm{C}$. The ordered $\mathrm{Pd}_{8} \mathrm{~W}$ phase is stable, but to this day does not appear in the equilibrium Pd-W phase diagram. An example of this phenomenon is shown in Fig. 2.3. Other examples of isostructural $\mathrm{Pd}_{8} \mathrm{X}$ phases induced by irradiation are $\mathrm{Pd}_{8} \mathrm{Mo}$ [17] and $\mathrm{Pd}_{8} \mathrm{~V}$ [18]. Another well-known example of RISR over short length scales is the amorphization of crystalline alloys, especially ordered intermetallic compounds. Lesueur [19] reported the first observation of radiation-induced amorphization of a neutron-irradiated Pd-Si alloy, and Thomas et al. [20] later observed a similar result in near-equiatomic NiTi alloys irradiated by high-voltage electrons. Numerous examples of radiation-induced amorphization have since been observed in many different ordered intermetallic compounds under a wide variety of bombarding species and irradiation temperatures. The third manifestation of RISR is the crystallization of an amorphous alloy. Early examples were reported by Parsons and Balluffi [21] and Azam et al. [22] and similar findings have been reported in many other amorphous alloys. The literature on RISR over short length scales is therefore quite large, so to keep the size of this review manageable, we will keep the focus on solute redistribution over longer diffusive length scales.
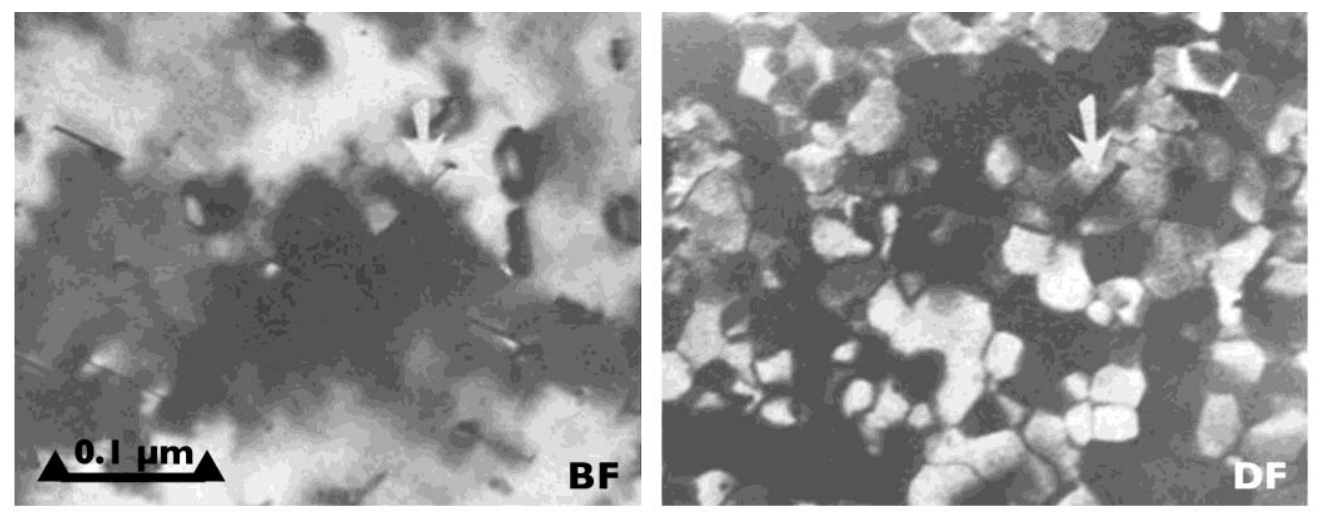

Figure 2.3. Bright field (BF) and dark field (DF) TEM micrographs of a Pd-18\%W sample 
irradiated with $400 \mathrm{KeV}$ protons to a dose of $1.7 \mathrm{dpa}$ at $600{ }^{\circ} \mathrm{C}$ then aged for 4 weeks at $600{ }^{\circ} \mathrm{C}$ [16]. The arrows show the same region in each micrograph. The DF image was taken using a superlattice reflection.

The earliest observations of Okamoto and Wiedersich [9] have been augmented by many other observations of RIS. Their conjecture of a preferential association between undersized atoms and radiation-produced interstitials is by now indisputable. It was noted as long ago as 1983 by Rehn and Okamoto [23] that the depletion of an undersized solute at a point defect sink had never been observed; we believe that this is still the case today. It is also true, for the most part, that oversized solute atoms tend to become depleted at point defect sinks as a consequence of radiation damage. The most notable exception to this behavior in fcc systems are the observations the segregation of oversized Ge atoms to interstitial dislocation loops and voids [24] and the segregation of Ge to the free surface [23] of irradiated undersaturated Ni-Ge alloys. These experiments resulted in the formation of $\mathrm{Ni}_{3} \mathrm{Ge}$ precipitates $\left(\mathrm{Ni}_{3} \mathrm{Ge}\right.$ is isostructural with $\mathrm{Ni}_{3} \mathrm{Si}$ ) at the specified point defect sinks. Gupta and Lam [25] explained these observations by calculating the binding energy of mixed Ni-Ge dumbbell interstitial complexes and showing that it is large enough to enable the segregation of Ge by interstitial diffusion, analogous to the behavior of Ni-Si complexes.

Undersized solutes in bcc alloys also segregate to point-defect sinks. Chakin et al. [26] reported the segragation of Fe to free surfaces in a $\mathrm{Cr}-35 \mathrm{wt} \% \mathrm{Fe}$ alloy neutron-irradiated at 750 ${ }^{\circ} \mathrm{C}$ (Fe is undersized in Fe-Cr alloys [27]), while Erck and Rehn [28] showed that Re segregates to free surfaces in several ion-irradiated Mo-Re alloys, in which Re is reported to be undersized according to the atomic volumes tabulated by King [29]. Meslin et al. [30] used APT to observe the clustering of $\mathrm{Mn}$ at unidentified defects in a model $\alpha$-Fe alloy containing 1 at $\% \mathrm{Mn}$ irradiated by $10 \mathrm{MeV}$ Fe ions at $400{ }^{\circ} \mathrm{C}$ to a dose of $0.5 \mathrm{dpa}$. Wakai et al. [31] found RIS of Cr to dislocation loops in several binary $\mathrm{Fe}-\mathrm{Cr}$ alloys electron irradiated at temperatures ranging from 300 to $700{ }^{\circ} \mathrm{C}$, while Bhattacharya et al. [32] observed RIS of $\mathrm{Cr}$ to dislocation loops in 3 binary $\mathrm{Fe}-\mathrm{Cr}$ alloys self-ion irradiated at $400{ }^{\circ} \mathrm{C}$. Interestingly, both $\mathrm{Mn}$ and $\mathrm{Cr}$ are slightly oversized [27,33], so while the findings in Ni-Ge alloys appear to be unique in fcc systems, bcc alloys clearly behave differently. Meslin et al. [30] suggest that Mn interstitials are dragged by mobile point-defect clusters, while Bhattaharya et al. [32] invoke coupling between the point-defect and $\mathrm{Cr}$ fluxes to their point-defect sinks. However, the operation of other mechanisms cannot be 
excluded.

The examples of RIS discussed to this point are explained by the coupling or binding of solute atoms and interstitials. Well over four decades ago Aust et al. [34] recognized that the binding of vacancies to substitutional solute atoms could produce solute segregation to grain boundaries (GBs), producing signficant hardening. In this case excess vacancies were produced by rapid quenching from a high temperature, not by irradiation. This phenomenon, which is now called thermal non-equilibrium solute segregation (TNES), was invoked by Aust et al. [34] to explain the results of Westbrook and Aust [35], who observed significant increases in microhardness near GBs in bicrystals of $\mathrm{Pb}$ doped with $\mathrm{Sn}, \mathrm{Ag}$, $\mathrm{Au}$ or In and air-cooled from temperatures in the range 200 to $300^{\circ} \mathrm{C}$. TNES has since been observed in many materials, including austenitic and ferritic steels. A representative concentration profile for the TNES of $\mathrm{Cr}$, taken from the work of Goodwin et al. [36], is shown in Fig. 2.4. The steel is type 304, which was solution treated at $1050{ }^{\circ} \mathrm{C}$, cooled at $\sim 20^{\circ} \mathrm{C} / \mathrm{s}$ and examined by analytical electron microscopy. The data on this unirradiated specimen show that the concentration of $\mathrm{Cr}$ at grain boundaries is $\sim 7 \mathrm{wt}$. \% larger than in the bulk.

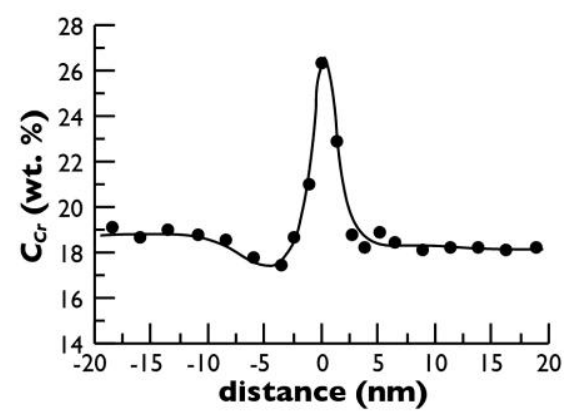

Figure 2.4. Thermal non-equilibrium segregation (TNES) of $\mathrm{Cr}$ to a grain boundary in a Type 304 stainless steel cooled from $1050^{\circ} \mathrm{C}$ to room temperature at $\sim 20^{\circ} \mathrm{C} / \mathrm{s}$. Data of Goodwin et al. [36].

Nearly concurrently with the work of Aust, Westbrook and co-workers [34,35], Howard and Lidiard [37] realized that the flow of excess vacancies to an excellent point-defect sink, like a general GB, could actually produce a deficiency of solute provided that the solute atoms diffused more rapidly than the host solvent atoms. In other words, a flux of vacancies toward the GB could induce a flow of solute atoms in the opposite direction; strong binding between solute atoms and vacancies was not needed to induce solute segregation at sinks. Indeed, Anthony and Hanneman [38] proposed that solute depletion could occur at GBs or free surfaces even in the face of positive binding between solute atoms and vacancies. Anthony [39] developed this idea 
quantitatively and demonstrated its consequences in selected physical situations. Marwick [40] was apparently the first to recognize that solute depletion by the flow of vacancies toward pointdefect sinks could produce solute segregation during irradiation; he called this the "Inverse Kirkendall Effect", now known generally as the IKE.

The IKE in a binary A-B alloys is illustrated schematically in Fig. 2.5. In this example A atoms diffuse faster than B atoms, hence the flux of vacancies towards the point-defect sink induces a preferential flux of $\mathrm{A}$ atoms in the opposite direction. The sink becomes depleted in $\mathrm{A}$ atoms and since the overall concentration of $\mathrm{A}+\mathrm{B}$ atoms must remain constant, the sink becomes enriched in B atoms.

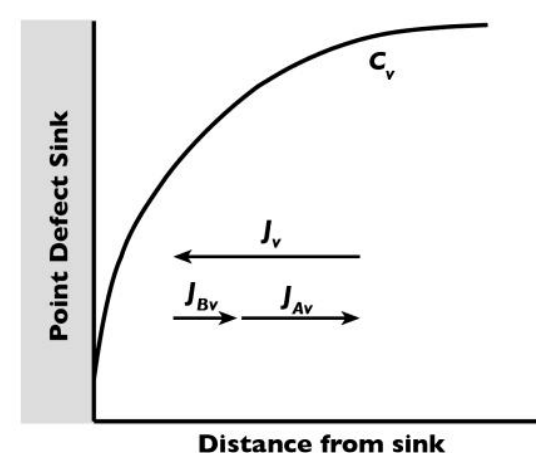

Figure 2.5. Schematic diagram illustrating the fluxes of vacancies and atoms in the inverse Kirkendall effect.

The prescience of Anthony and Hanneman [38] regarding RISR is also illustrated in Fig. 2.6(a), where their schematic composition profiles at a grain boundary are compared with real composition profiles of $\mathrm{Ni}$ and $\mathrm{Fe}$ in a neutron-irradiated steel [41]. The profiles published by Anthony and Hanneman [38] preceded the discovery of RIS. Nevertheless, they bear a remarkable resemblance to the composition profiles reported by Norris et al. [41] in Fig. 2.6(b). We emphasize here that these similarities do not imply that the experimental concentration profiles were created by the IKE. The main point is that profiles like these can arise through different segregation mechanisms, and that the mechanism cannot be inferred simply from the profiles themselves. 


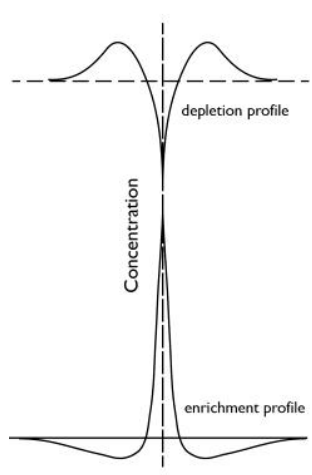

(a)

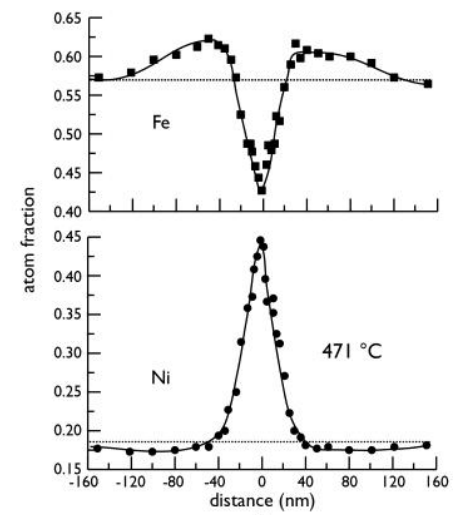

(b)

Figure 2.6. (a) Schematic concentration profiles freely adapted from the drawing published by Anthony and Hanneman [38] at a GB in a quenched alloy; (b) RIS induced concentration profiles measured by Norris et al. [41] illustrating the depletion of $\mathrm{Fe}$ and enrichment of $\mathrm{Ni}$ at a grain boundary in a stainless steel irradiated by neutrons at $471^{\circ} \mathrm{C}$.

Modeling, both at the continuum scale and at the atomic scale, has been widely used to reproduce and predict segregation profiles, and to identify segregation mechanisms. This modeling requires the calculation of the coupling between point defect fluxes and atomic fluxes, as illustrated in Fig. 2.5 in the case of IKE. The quantitative determination of this coupling remains a challenge, especially in non-dilute alloys, as migrating point defects sample a complex configurational space, influencing the redistribution of chemical species. Research on these issues continues to this day, and involves a combination of theory, atomistic calculations, Monte Carlo simulations and phase-field modeling. The ultimate objective is to inform the design of radiation-damage resistant materials over the vast range of length scales from the atomic, through the nano-meter and micro-meter microstructural scales to the design stage. In the remainder of this review article we focus on the progress in theory, modeling and experiments, emphasizing advances in understanding of RIS down to the smallest of length scales.

\section{Diffusion under irradiation conditions}

Point defects are continuously created under irradiation, producing defect supersaturations. A first consequence of these supersaturations is that thermally activated diffusion of chemical species is accelerated, a phenomenon referred to as radiation-enhanced diffusion. A second consequence, already noted, is that the supersaturations produce net permanent fluxes of point defects to sinks. As illustrated in Fig. 2.5, the coupling between the diffusion of point defects and chemical species can lead to solute redistribution. Such coupling 
can be very complex, especially in concentrated alloys. The first quantitative models of coupling were thus mostly concerned with dilute alloys, where a rather small set of atomic configurations and jump frequencies is sufficient to capture the main physical effects, as illustrated below.

In dilute alloys, solute redistribution occurs by the motion of vacancy-solute and/or interstitial-solute complexes. To simplify subsequent discussion, complexes involving vacancies and solute or solvent atoms will be referred to as V-S complexes. Their interstitial counterparts will be called I-S complexes. Solute redistribution can occur via the migration of these complexes, provided that their dissociation energies are much larger than their migration energies. The migration of V-S and I-S complexes is disarmingly difficult to quantify. We present some relevant examples to illustrate the difficulties.

An early attempt at describing the motion of a V-S complex in a dilute binary alloy is shown in Fig. 3.1(a), adapted from Wiedersich and Lam [42]. Assuming that the interactions between a solute atom and a vacancy are restricted to the first nearest neighbor shell, there are only five distinct jump frequencies. These jump frequencies, indicated by the $\omega_{i}(i=0$ to 4$)$, differ whenever the nearest neighbor atoms or vacancies differ (the numberings are consistent with the descriptions of Howard and Lidiard [37] and Howard and Manning [43]). For example, the atoms with jump freqencies $\omega_{1}$ and $\omega_{4}$ are both nearest neighbors of the oversized solute atom (black), but their other nearest neighbors are different, so their $\omega_{i}$ into the neighboring vacant sites differ from each other and all the other atoms. Diffusion in this model is expressed in terms of ratios of the calculated jump frequencies, which depend on the V-S binding energy. In fcc dilute alloys the diffusion of solute atoms can be explained using the 5 jump frequencies depicted in Fig. 3.1(a). This becomes inadequate when the solute concentration increases, as illustrated in Fig. 3.1(b). The insertion of only 2 more oversized solute atoms changes the jump frequencies of many of the atoms neighboring the vacancies, designated as ${ }_{i}^{*}$, increasing the number of jump frequencies needed to model diffusion. The added complexity illustrates the need for new approaches to modeling diffusion in concentrated alloys. 


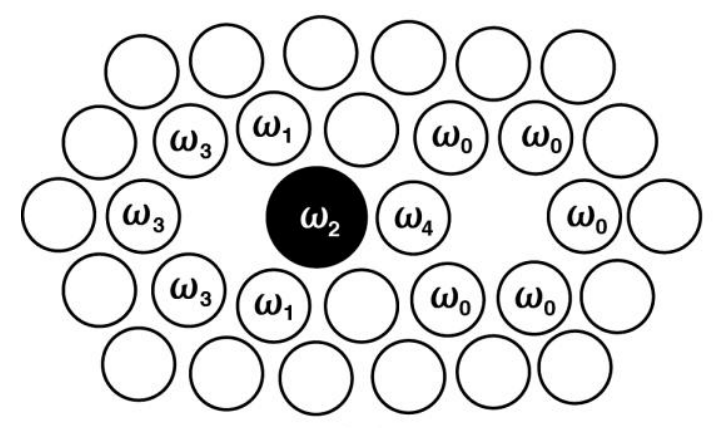

(a)

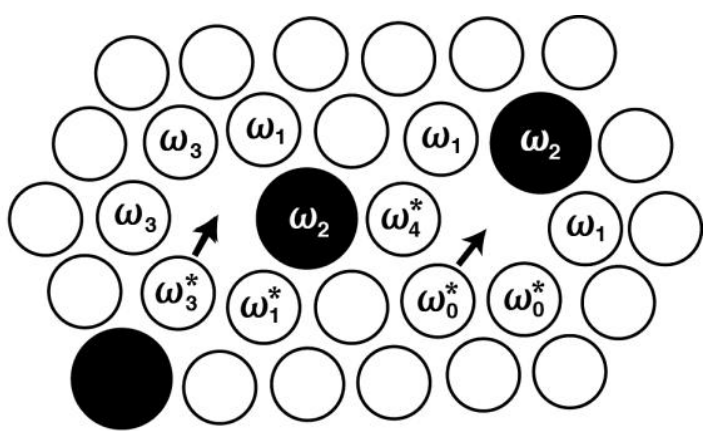

(b)

Figure 3.1. (a) The atom configurations around two vacancies in a dilute substitutional alloy ( $3 \%$ solute) containing an oversized solute atom (black). The jump frequencies, $\mathrm{W}_{\mathrm{i}}$, of the atoms are labelled differently to emphasize that their jump frequencies into the vacant lattice site are different and influenced by their local surroundings. The diagram is adapted from Wiedersich and Lam [42]; (b) Illustrating the additional jump frequencies associated with atoms in concentrated alloys containing about $10 \%$ oversized solutes. The jumps indicated by the arrows are affected because the second nearest neighbors of the atoms are different in (a) and (b).

The diffusion of interstitials is also much more difficult to quantify when the solutions are concentrated than when they are dilute. Consider the example of the motion of an I-S complex shown in Fig. 3.2, also taken from the article by Wiedersich and Lam [42]. This figure describes the possible jumps of an interstitial atom from one lattice position to another in a dilute alloy. The interstitial atom is part of an I-S complex in the so-called mixed dumbbell configuration, which is depicted in Fig. 3.2 as a solute-solvent atom pair sharing a lattice site. We emphasize that the formation of mixed dumbbells is possible only when self-interstitials have been introduced into the crystal by irradiation; the formation of I-S complexes under thermal equilibrium conditions is essentially impossible. In the example shown in Fig. 3.2, the solute atom migrates from the upper left position to the lower right position by rotation of the mixed dumbbell. The configuration shown in Fig. 3.2 is not unique; other possible configurations for the migration of mixed dumbbells are discussed by Wiedersich and Lam [42] and Robrock [44]. Diffusion of undersized interstitial solute atoms in irradiated dilute face-centered cubic alloys is quite complicated to predict theoretically, even for mixed dumbbells in $\langle 100\rangle$ orientation. Approximate solutions can be found in papers by Johnson and Lam [45] and Barbu [46]. In concentrated solid solutions, even those exceeding 1 at. \% or so, the configurations depicted in Fig. 3.2 are unrealistic for a variety of reasons: I-S complexes can have other I-S complexes as nearest neighbors; I-S complexes can be replaced by solute-solute atom 
complexes; both types of interstitial atom complexes can can have more than one solute atom, or even vacancies as nearest neighbors. It goes without saying that the number of jump frequencies that must be accounted for in any theory increases dramatically as the solute concentration increases.
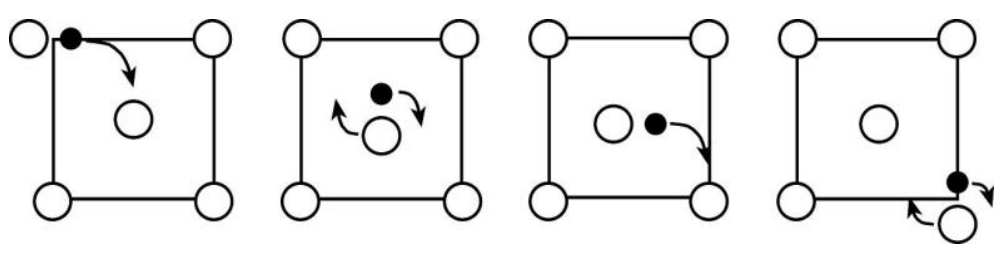

Figure 3.2. Illustration of the diffusion of a mixed dumbbell interstitial by rotation inside the unit cell, followed by exchange of the undersized solute atom (small filled circle) with a neighboring solvent atom (larger open circles). The diagram is adapted from Wiedersich and Lam [42].

The relatively straightforward discussion involving the complications inherent in describing the diffusive transport of atoms in concentrated solid solutions illustrates the difficulties in formulating theories of diffusion in irradiated alloys. We have so far considered only the complications associated with jump frequencies. Another important factor that must be considered is the correlation associated with various jumps from one lattice configuration to another. Simply put, correlation in the context of diffusion describes the probability that an atom or complex will exchange positions with a new site instead of returning to the site or configuration from which it has already jumped. In diffusion by the vacancy mechanism in pure metals an atom that has jumped from one lattice site to another is most likely to jump right back into its previous site, everything else being equal. This is not so for the vacancy with which the atom exchanged places, since the vacancy in an fcc crystal has 12 nearest neighbor positions to choose from. The quantitative impact of correlation in diffusion is described the the "correlation factor" or "correlation coefficient", which is usually designated by the symbol $f$. The magnitude of $f$ depends on the mechanism of diffusion and the underlying crystalline lattice [47-49] and always ranges in value from 0 to 1 . If the diffusion of a given atomic species is uncorrelated, its value of $f=1$; in self-diffusion in a pure metal the motion of vacancies is uncorrelated. If $f=0$ an atom will always exchange positions with the same vacancy and will never migrate.

One other factor affects diffusion by the vacancy mechanism. It is the so-called "vacancy wind factor" introduced by Manning [50] and generally designated by the symbol $S$. The vacancy wind affects diffusion only in solid solutions and exists primarily in response to a vacancy flux. This causes each atom to have a slightly increased probability of jumping in a 
direction opposite to the vacancy flux, i.e. in the direction in which the local concentration of vacancies is larger. As might be expected, $S$ is dependent on $f$, and under some conditions the influence of $S$ on diffusion is not negligible.

Deriving theories of diffusion, taking into account the formidable landscapes for the transport of atoms in concentrated solutions, is clearly a daunting task. Since the number of independent jump frequencies that contribute to macroscopic transport increases dramatically with the number of solute atoms surrounding a diffusing atom, it becomes more advantageous to consider kinetic models that average out the effect of the surrounding solute atoms. Significant progress has been made recently using the self-consistent mean-field (SCMF) kinetic formalism introduced by Nastar et al. [51-53], which was recently reviewed by Nastar and Soisson [2]. The introduction section in the paper by Nastar et al. [51] is particularly helpful in documenting the history of earlier theories of diffusion leading up to the SCMF theory. This formalism starts from a general expression for the fluxes of chemical species and point defects as linear combinations of the driving forces, i.e., the gradients of the chemical potentials. Owing to the kinetic coupling between the migration of all these species, the coefficients relating fluxes of species "i" to forces of species " $\mathrm{j}$ " are expressed in terms of the jump frequencies that determine the probabilities of a given transition from one lattice site to another. An expression for these macroscopic kinetic coefficients is derived in terms of the atomistic-scale thermokinetic parameters of the alloy of interest. This derivation relies on the use of effective atomic interactions that capture the correlations in the coupled migration of the different species.

A detailed presentation of the SCMF method is beyond the scope of the present review. It is uesful to note however that three different types of approximation are used in that approach. The first approximation concerns the energetics: the internal energy of a microscopic configuration is expressed as a sum of cluster interaction energies weighted by the occurrence of each cluster; in the simplest approximation, these interactions are restricted to pairs, but triplet and tetrahedron interactions can be included as well, especially since they can now be routinely extracted from first principles calculations. The second type of approximation pertains to the calculation of configurational entropy: the infinite series of correlation functions of lattice site occupation needs to be truncated to yield closed-form expressions. Truncation beyond the onesite, two-site, three-site...correlations corresponds to the so-called point, pair, triplet approximations; this helps clarify the terminology frequently used in publications that invoke the 
SCMF method. The cluster variation method provides a general formalism for generating and implementing these approximations [54]. We note that these first two types of approximation involving energetics and statistics are characteristic of mean-field models. The third type of approximation introduced in the SCMF are so-called effective interactions that take into account the fact that, in the presence of a non-zero net flux, the probabilities of finding given pairs, triplets,... of diffusing species deviate from their equilibrium values. These deviations originate from the correlated migration of atoms and point defects, and thus the effective interactions capture these correlations. Pairwise effective interactions are often used for simplicity since they often provide results that compare very well with full Monte Carlo simulations, but higher order effective interactions can be employed as well. One last important point concerns the range of these effective interactions. As described for instance by Garnier et al. [55], this range can be physically related to the various shells within which correlation effects are taken into account. The SCMF approach, which was originally derived for vacancy-mediated atomic transport, has been extended to interstitial migration [56,57], and recently, to include stress effects [58-60]. We discuss the impact of these diffusion models on RIS in Sec. 4.

\section{Theories of RIS}

Multiple approaches have been employed over the years to model and predict RIS in dilute and concentrated alloys. Until the last decade or so, these models relied on simplified treatments of the alloying effects on correlation coefficients, e.g. the assumption $f=1$ in some models, but they were nevertheless able to capture important physical effects and to help understand experimental data. More recently, through advances in first principles computing of jump frequencies and in the development of mean-field kinetic theories such as the SCMF approach, new RIS models have been aiming for quantitative and alloy-specific predictions. We

first review an important example of the earlier models and its application to RIS in Ni-Si alloys. We then highlight some of the recent developments, in particular for austenitic, ferritic and ferritic/martensitic steels.

\subsection{Mean-Field Rate-Theory Models.}

The first important family of RIS models was built around a phenomenological description of diffusion in concentrated binary alloys at the continuum level using the random 
alloy model of Manning [61]. In Manning's model, diffusion of chemical species A and B is characterized by two distinct jump frequencies, one for each type of atom, and these frequencies are assumed to be independent of their local ennvironments. The most important early RIS model built on this assumption is the rate-theory model introduced by Wiedersich et al. [62] Other models in this class, including those of Marwick [40], English et al. [63] and Wolfer [64], who used an approach based on the thermodynamics of irreversible processes. A related, but alternative approach was proposed by Grandjean et al. [65]; it included the effect of local chemical environments on jump frequencies by using a mean-field model on a lattice, with a point approximation for the configurational entropy [66].

Due to its importance and early success in capturing the details of RIS in Ni-Si alloys, we describe here the theory of Wiedersich et al. [62] (the WOL theory) of RIS in concentrated alloys. Their model is an extension of the original kinetic rate theory of Johnson and Lam [45], which is valid only for dilute solid solutions and utilizes models for diffusion of point-defect solute-atom complexes such as those depicted in Figs. 3.1 and 3.2. The Johnson-Lam model cannot be used to describe diffusion in concentrated solid solutions because the probabilities that vacancies or interstitials have only one solute atom as a nearest neighbor are very small, as illustrated in Fig. 3.1(b). The jump frequencies of the atoms neighboring a vacancy, as well as other parameters, e.g. $f$ for the individual atoms, can then no longer be estimated with any degree of accuracy. Likewise, when the concentration of undersized solute atom increases the number of solute-solute dumbbells will increase, so calculations of diffusion involving only the transport of mixed dumbbells become unrealistic.

To deal with the formidable problem of formulating a theory of diffusion involving the numerous types of point-defect solute-atom complexes, WOL introduced the idea of partial diffusion coefficients related to 4 different partial diffusivity coefficients in a binary alloy. The partial diffusivity coefficients are defined by the equations

$$
d_{A, B / v, i}=\frac{1}{6} \omega_{A, B / v, i} b_{v, i}^{2} Z_{v, i},
$$

where the subscripts $d_{A, B /, i}$ represent partial diffusivity coefficients of A or B atoms, respectively, by interchange with vacancies (v) or interstitials (i); these are identical to the partial diffusivity coefficients of vacancies or interstitials by interchange with A or B atoms. The quantities $b_{v, i}$ and $Z_{v, i}$ are the jump distances and coordination numbers, respectively, of vacancies and interstitials, 
which can differ in principle. The important quantities in eqns. (1) are the $\omega_{A, B / v, i}$, which represent the effective jump frequencies with which A or B atoms exchange places with vacancies or interstitials. These equations are readily extended to multi-component alloys [67] and have temperature dependencies of the Arrhenius type, with activation energies that can be very difficult to estimate because the point defects and solute atoms can be bound to, or repelled from the individual atoms, or groups of atoms, in the crystal. Additionally, as indicated earlier, these energies (hence jump frequencies) should depend on the configuration of the solute-atompoint-defect complex and on the composition of the alloy.

The WOL theory next prescribes equations for the fluxes of the various components in the alloy system, including not only the atomic species, but also the vacancies and interstitials. This is done by first defining partial diffusion coefficients in terms of the partial diffusivity coefficients by the equations

$$
D_{A, B / v, i}=d_{A, B / v, i} f_{A, B / v, i} C_{v, i}
$$

and

$$
D_{v, i / A, B}=d_{A, B / v, i} f_{v, i / A, B} C_{A, B}
$$

where the concentrations $C_{v, i}$ and $C_{A, B}$ are atomic fractions and $f_{A, B / v, i}$ and $f_{v, i / A, B}$ are correlation coefficients for the jumps of A or B atoms into vacant or interstitial sites and the corresponding correlation coefficients for vacancy and interstitial jumps with A and B atoms, respectively. Note that $f_{A, B / v, i} \neq f_{v, i / A, B}$ but $d_{A, B / v, i}=d_{v, i / A, B}$. If the correlation coefficients are all equal to unity, the total diffusion coefficients for the atoms and point defects are given by the equations

$$
D_{A, B}=D_{A, B v}+D_{A, B i}
$$

and

$$
D_{v, i}=D_{v, i A}+D_{v, i B}
$$

The assumption $f_{A, B / v, i}=f_{v, i / A, B}=1$ is one of the major approximations of the theory of Wiedersich et al. [62].

The fully-developed WOL theory predicts that under irradiation conditions the fluxes of all the atomic species depend on the concentration gradients of both vacancies and interstitials, as well as their own gradients, and that the fluxes of point defects depend on the gradients of the atomic species as well as their own gradients. These are the most important distinctions between 
diffusion under irradiation and diffusion under normal conditions. With respect to a fixed lattice plane the fluxes of A atoms, B atoms, vacancies and interstitials are not independent, and must satisfy the equation

$$
J_{A}+J_{B}=-J_{v}+J_{i} .
$$

Wiedersich et al. [62] showed that under steady-state irradiation conditions

$$
\nabla C_{A}=\frac{C_{A} C_{B} d_{A i} d_{B i} \Omega}{\alpha\left(d_{A i} C_{A} D_{B}+d_{B i} C_{B} D_{A}\right)}\left\{\frac{d_{A v}}{d_{B v}}-\frac{d_{A i}}{d_{B i}}\right\} \nabla C_{v},
$$

where $\alpha$ is the thermodynamic factor. Equation (5) follows from the conditions $J_{A}=J_{B}=0$ and $J_{v}$ $=J_{i}$. In a coordinate system such as that in Fig. 2.5 the gradient of $C_{v}$ is always positive. According to eq. (5) the sign of the gradient of $C_{A}$ will therefore depend on the sign of term in curly brackets, which from eq. (1) depends on the ratios of the $\omega_{A, B / v, i}$. If the jump frequencies are such that $d_{A v} / d_{B v}>d_{A i} / d_{B i}$, the gradients of $\mathrm{A}$ atoms and vacancies will have the same sign. What happens physically is that if $\mathrm{A}$ and $\mathrm{B}$ interstitial atoms jump with approximately equal frequencies but $\mathrm{A}$ atoms exchange place with vacancies much faster than they do with $\mathrm{B}$ atoms, the diffusion of A atoms will be faster than the diffusion of B atoms. RIS at the point defect sink is thus realized by depletion of $\mathrm{A}$ atoms and enrichment of $\mathrm{B}$ atoms; this is essentially the IKE.

Examples of the utility of the WOL theory are demonstrated in Figs. 4.1 and 4.2, which show data of Averback et al. [68] on the growth rate of $\mathrm{Ni}_{3} \mathrm{Si}$ surface films in a $\mathrm{Ni} 12.7$ at. \% $\mathrm{Si}$ alloy. Averback et al. invoked the WOL theory to derive a model of film growth using several assumptions: 1. The near-surface region is rapidly depleted of Si as strongly-bound Si-interstitial complexes migrate towards the surface and form an $\mathrm{Ni}_{3} \mathrm{Si}$ film, similar to that seen in Fig. 2.2(c), when the solubility limit is reached; 2. Vacancies play an important role through their affect on recombination, but they play no part in diffusion due to the formation of stable Si I-S complexes; 3. A steady-state concentration profile is established in the bulk alloy behind the surface film, where the solute is depleted as the film thickens; 4 . The solid solution is taken as ideal (this is not mentioned by Averback et al., but is implicitly assumed. Using a simplified model of the diffusion geometry to calculate the flux of solute in the depleted zone behind the growing $\mathrm{Ni}_{3} \mathrm{Si}$ surface film, Okamoto et al. [69] had already predicted that the thickness of the surface film should increase linearly with the square root of the dose.

The data in Fig. 4.1, as well as other data of Averback et al. [68] (not shown), illustrate 
how well the predicted dose-dependence of the film thickness is observed at all irradiation temperatures. The growth rate, given by the slopes of the curves in Fig. 4.1, increases with increasing temperature up to about $565{ }^{\circ} \mathrm{C}$, then decreases such that the growth rate at $639{ }^{\circ} \mathrm{C}$ is actually lower than that at $452{ }^{\circ} \mathrm{C}$. Averback et al. [68] predict that the activation energy for growth at lower temperatures should be $E_{m v} / 4$, where $E_{m v}$ is the migration energy of vacancies, but at higher temperatures the growth rate should decrease with an Arrhenius-type temperature dependence controlled by the formation energy of vacancies, $E_{f v}$; specifically, at high temperatures the growth rate should vary as $\exp \left(E_{f v} / 2 k T\right)$. The theory also predicts that at lower temperatures the growth rate should increase with decreasing dose rate. The predicted temperature and dose-rate dependencies are displayed in Fig. 4.2. While the magnitudes of $E_{m v}$ and $E_{f v}$ are not known with certainty, the values implied by the results shown in Fig. 4.2, $E_{m v} \approx$ $1.2 \mathrm{eV}$ and $E_{f v} \approx 1.5 \mathrm{eV}$, are consistent with expectation. Additionally, the growth rates at dose rates of $2.6 \times 10^{-5} \mathrm{dpa} / \mathrm{s}$ are roughly a factor of 2 higher than those at a dose rate of $3.1 \times 10^{-4}$ $\mathrm{dpa} / \mathrm{s}$, which is also consistent with the predictions of Averback et al. [68].

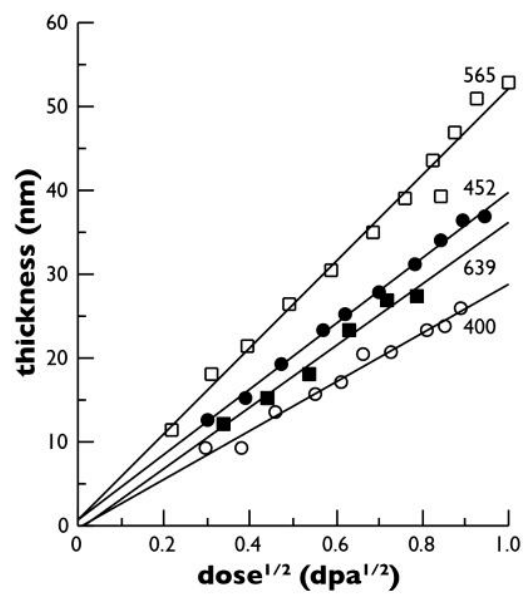

Figure 4.1. Plot of $\mathrm{Ni}_{3} \mathrm{Si}$ surface film thickness vs. square root of the dose as a function of temperature (in ${ }^{\circ} \mathrm{C}$, shown next to each curve) in a Ni-12.7 at.\% Si alloy irradiated by $2 \mathrm{MeV}$ $\mathrm{He}^{+}$ions at a dose rate of $2.6 \times 10^{-5} \mathrm{dpa} / \mathrm{s}$. The plot is adapted from Fig. 6 in the paper by Averback et al. [68] 


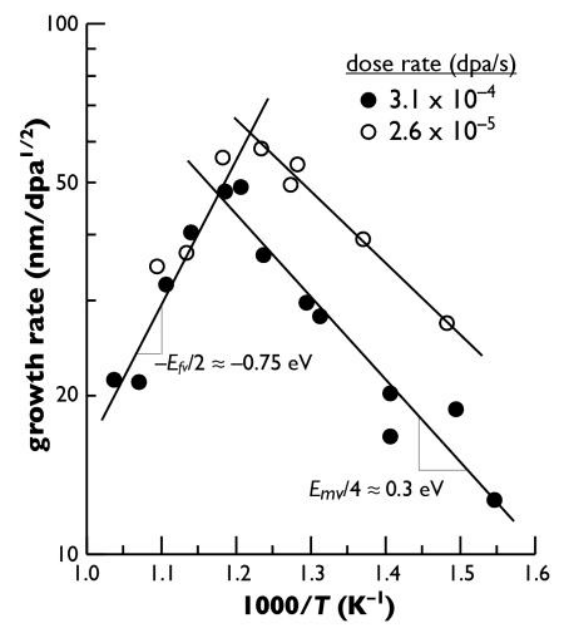

Figure 4.2. Arrhenius plots of growth rates of the $\mathrm{Ni}_{3} \mathrm{Si}$ films for the 2 dose rates shown. The parameters $E_{f v}$ and $E_{m v}$ are defined in the text. Data of Averback et al. [68].

The physical reasons for the observed temperature dependencies involve the dominant role played by recombination. The rate of recombination will always increase with an increase in the vacancy concentration. In solids irradiated at a fixed dose rate $C_{v}$ passes through a minimum value at temperatures between 0.3 and $0.5 T_{m}$. At high temperatures $C_{v}$ is controlled by the thermal equilubrium concentration, which decreases with decreasing temperature. When $T<$ $0.3 T_{m} C_{v}$ will decrease with increasing $T$ because vacancies will migrate more rapidly to sinks (vacancy motion is always thermally activated, with activation energy $E_{m v}$, and increases with increasing $T$ ). The rate of RIS is inversely related to $C_{v}$, hence must pass through a maximum at temperatures corresponding to the minimum in $C_{v}$. Assuming that the concentration of Si I-S complexes is unaffected by temperature, the maxima of growth rate on $T$ seen in Fig. 4.2. are consistent with the temperature dependencies of RIS, depending on the vacancy formation energy for vacancies, $E_{f v}$, at high temperatures and on vacancy motion, hence $E_{m v}$, at low temperatures. The lower growth rates associated with higher dose rates, observed at low temperatures, are also consistent with the larger vacancy concentrations produced, leading to increased recombination, thereby reducing the concentration of I-S complexes and rate of RIS.

\subsection{Approaches Utilizing the Onsager Formalism}

The self-consistent mean-field (SCMF) theory of Nastar and coworkers [51-53] was discussed briefly in Sec. 3 as a promising approach to modeling diffusion in concentrated solidsolution alloys and their behavior under irradiation conditions. We noted that the starting point 
was the relationship between the fluxes of the component species and the driving forces compelling their transport, namely the gradients of their chemical potentials. Complexity arises from the fact that the fluxes of all the species in the alloy are potentially influenced by the chemical-potential gradients of all the other species. The fluxes of the species in a binary A-B alloy under irradiation conditions (atoms A, B, vacancies and interstitials) in eq. (4) are written in terms of the Onsager formalism. Following the procedure presented by Wolfer [64] we first recognize that there are 2 contributions to $J_{A}$ and $J_{B}$, specifically

$$
J_{A}=J_{A v}+J_{A i}
$$

and

$$
J_{B}=J_{B v}+J_{B i},
$$

where the fluxes $J_{A v}, J_{A i}, J_{B v}$, and $J_{B i}$ take the contributions of both interstitials and vacancies to the fluxes of both species into account. Of course, in the absence of irradiation there are no contributions from the interstitial fluxes. For consistency with eq. (4) the vacancy and interstitial fluxes must also satisfy the equations

$$
-J_{v}=J_{A v}+J_{B v}
$$

and

$$
J_{i}=J_{A i}+J_{B i}
$$

In the Onsager formalism the fluxes of $\mathrm{A}$ and $\mathrm{B}$ atoms from the contributions of vacancies and interstitials, respectively, are represented by the equations

$$
J_{m v}=-\sum_{n} \frac{L_{m n}^{v}}{k_{B} T} \nabla\left(\mu_{n}-\mu_{v}\right)
$$

and

$$
J_{m i}=-\sum_{n} \frac{L_{m n}^{i}}{k_{B} T} \nabla\left(\mu_{n}+\mu_{i}\right),
$$

where the index $m$ represents the atomic species, $\mu_{m}, \mu_{v}$ and $\mu_{i}$ are the chemical potentials of species A, B, v and i, $k_{B}$ is Boltzmann's constant and $L_{m n}^{v, i}$ are the so-called Onsager coefficients, which are material parameters that represent the involvement of vacancies and interstitials in the transport of A and B atoms. Equations (8) show that the transport of A atoms is influenced not 
only by vacancies and interstitials, but also by B atoms and their chemical potential gradients; the same is true for the transport of B atoms.

Equations (8) are actually valid for multicomponent systems, with $m$ representing atoms $\mathrm{A}, \mathrm{B}, \mathrm{C} \ldots$ and the index $n$ representing a sum over the equivalent number of atoms; in a binary alloy there are only 2 terms in each sum in eqs. (8). The Onsager coefficients are directly related to diffusion coefficients, as one can show starting from eqs. (8) that the diffusion coefficient matrix is the product of the Onsager matrix by the so-called susceptibility matrix, whose coefficients are the second derivatives of the free energy with respect to concentrations [70]. Detailed relationships between Onsager coefficients and diffusion coefficients can be found, for instance, in the paper by Wolfer [64].

In concentrated alloys the prediction of diffusion geometries for all the components using the Onsager formalism remains a highly challenging problem. The main reason for this is that there are no direct data on the Onsager coefficients, so they must be either indirectly determined from diffusion experiments or calculated using atomistic simulations and then tested by comparing predicted with either experimental or computationally generated concentration profiles. The potential of the SCMF approach is that these Onsager coefficients can be reexpressed in terms of effective atomic interactions which result from correlation effects. By limiting the nature and range of these effective interactions, for instance to pairs of first nearest neighbors, one can obtained a self-consistent, closed-form expression for these effective interactions, and thus derive expression of the Onsager coefficients.

This is illustrated here for the diffusion of dumbbell interstitial atoms in a model A-B binary alloy [53,57]. The SCMF model, here with effective interactions restricted to pairs of first nearest neighbors, provides analytical expressions for the correlation factors; these are shown in Fig. 4.3, where it is seen that they are in excellent agreement with Monte Carlo simulations over the whole composition range. The significance of the information in Fig. 4.3 is that the correlation coefficients can be significantly different from unity and can vary with composition, contrary to the assumption made in the WOL model. The interested reader is advised to read Refs. [53,57] for exact definitions of the correlation coefficients and details of their calculation. Accurate values of the correlation coefficients are clearly needed for the quantitative modeling of RIS. The SCMF approach has been applied to several specific alloy systems such as Ni-Si [58,60], and dilute bcc Fe-X (X= Cr, Cu, Mn, Ni, P, Si) alloys [71]. As seen in Fig. 4.4, the 
inverse Kirkendall effect is predicted to result in Cr depletion at all temperatures, while the other solutes become enriched at defect sinks through solute drag at moderate irradiation temperatures. This work also illustrates that, in the case of dilute alloys, combining first principles calculations with the SCMF formalism makes it possible to directly calculate the partial diffusivity coefficients and the partial diffusion coefficients defined in eqs. (1) and (2) for alloy systems of interest. In the spirit of the Materials Genome Initiative, this approach therefore provides an extension of the WOL model that can be used for pre-screening alloy systems and thus reducing the number of experiments. The full realization of this approach will require an extension to concentrated alloys, which will be discussed at the end of this section.

The SCMF approach has also been extended to include stress effects on transport coefficients [58-60]. This work has uncovered unexpected coupling between solute transports and stress in dilute $\mathrm{Ni}-\mathrm{Si}$ alloys subjected to a volume-conserving uniaxial strain along one of the cube directions. In this alloy density-functional-theory (DFT) calculations indicate that the $\mathrm{Si}$ solute binds to the vacancy with an attractive binding energy $\approx 0.1 \mathrm{eV}$, and one therefore expects that at low temperatures, where this binding dominates, the vacancies will drag the Si solute atoms. At high enough temperatures compared to the binding energy, one would expect that the solute-vacancy complexes would no longer be stable, and that the solute atoms would then flow in a direction opposed to that of the vacancy flow. This behavior is indeed predicted by the SCMF model, as seen in Fig. 4.5, which displays the so-called solute-drag factor $L_{S i V} / L_{S i S i}$, the transition temperature between these two regimes is around $1000 \mathrm{~K}$, which is consistent with the Si-vacancy binding energy of $\approx 0.1 \mathrm{eV}$. The sign of the solute-drag factor, which can be positive or negative, drives solute transport in response to the flow of vacancies (Anthony has written an excellent review of the general phenomenon of vacancy wind effects [72]). Lastly, one would need to extend these results to the case of interstitial-mediated transport in order to compare the SCMF predictions for the Ni-Si alloy with the experimental results reported in Figs. 4.1 and 4.2.

Stress can modify atomic jump frequencies, and thus the Onsager transport coefficients, and one can thus anticipate that this would be the case for the transition temperature between the solute-drag IKE regimes as well. The remarkable prediction, however, is that the effect is much more complex and depends on the relative orientation of diffusion with respect to the applied strains (or stresses). In the case of a volume-conserving uniaxial strain, for instance, the transition temperature is shifted to lower and higher values for diffusion along and perpendicular 
to the tetragonal axis, respectively, as demonstrated on Fig. 4.5. Consequently, in the temperature regime labeled $\Delta T$, vacancies should drag solute atoms in the direction parallel to the uniaxial strain, while moving them in the direction opposed to the vacancy flux in the directions normal to this direction. It has been anticipated that such effects could lead to complex and intriguing solute flows near dislocation cores [58]. It is interesting to note that in the case of pure Ni, DFT calculations yielded an effect of stress on diffusion anisotropy in excellent agreement with the model of Ardell and Prikhodko [73]. The work of Garnier et al. [58-60] makes it possible to extend such investigations to the case of solute diffusion in alloys.

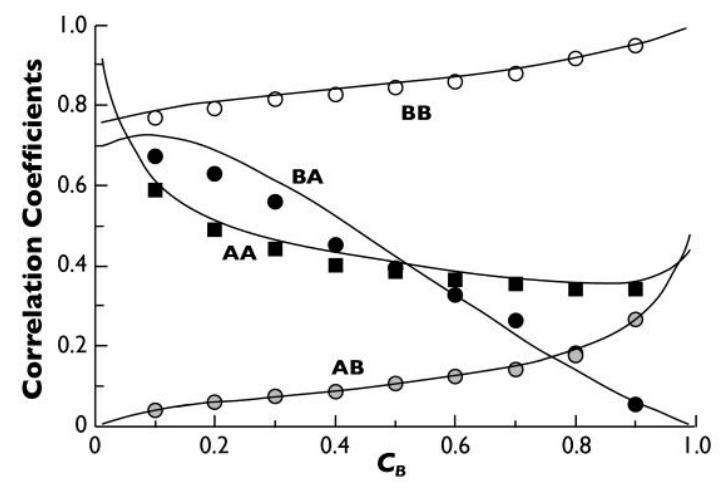

Figure 4.3. Correlation coefficients as a function of the concentration of $\mathrm{B}$ atoms, $C_{B}$, for a model A-B binary alloy with interactions between AA dumbbell interstitials and B solute atoms. The symbols are values directly calculated from Monte Carlo simulations, while the solid lines are the expressions obtained from the SCMF in the first-shell approximation for the effective interactions. The four curves labelled $\mathrm{A} / \mathrm{B}$ correspond to the correlation factors entering the flux equations for $\mathrm{A}(\mathrm{B})$ driven by the gradient of $\mathrm{B}(\mathrm{A})$. From the calculations of Nastar and Barbe $[53,57]$.

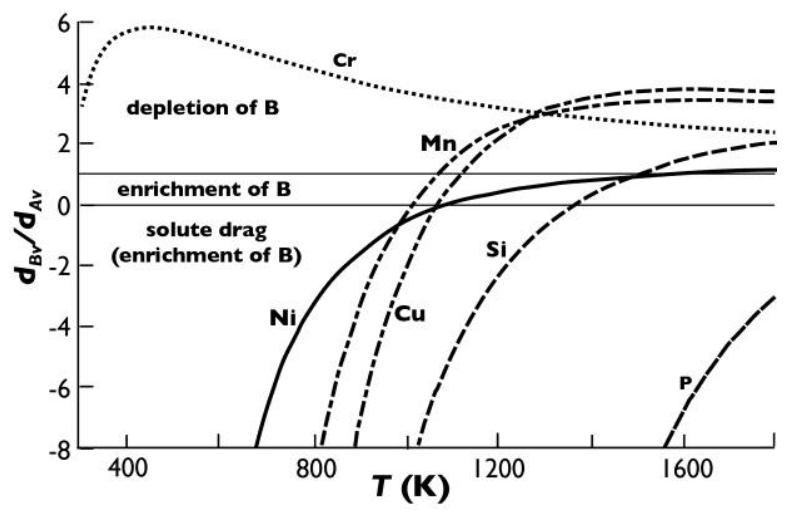

Figure 4.4. Ratio of partial diffusivities for various solutes in bcc Fe as a function of temperature. Radiation-induced depletion of $\mathrm{Cr}$ at sinks is expected for all temperatures due to the inverse Kirkendall effect $\left(d_{B v} / d_{A v}>1\right)$, while other solute atoms should segregate at sinks at low temperatures via a solute drag mechanism. From the calculations of Messina et al. [71]. 
In addition to advances in the modeling of coupled diffusion in multicomponent systems, illustrated in the previous paragraphs, the modeling of RIS has greately benefited from the maturation and availability of first-principles calculations. In particular it is now fairly straightforward to calculate accurately formation energies of defects, binding energies of solute atoms and defects, and atom-defect jump frequencies using quantum-mechanical approaches, e.g., based on DFT calculations [70,74,75]. Current computing limitations restrict these calculations to a rather small set of jump frequencies, typically tens to hundreds of frequencies, but this is sufficient to parameterize the diffusion models discussed in Section 3.

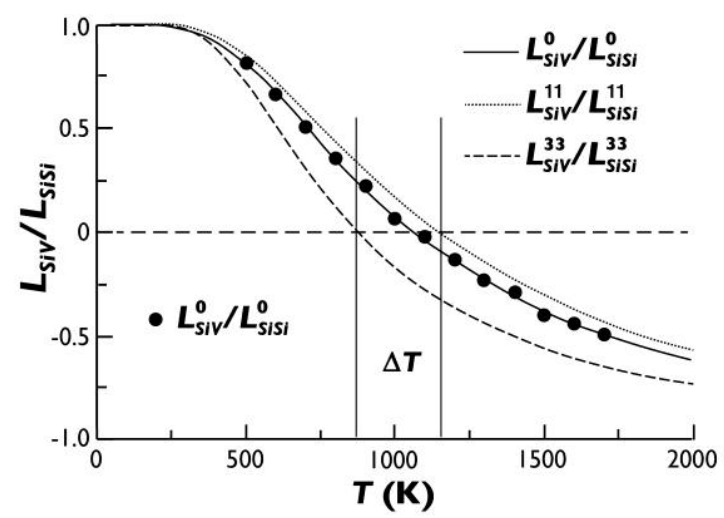

Figure 4.5. Ratio of Onsager coefficients $L_{S i v} / L_{S i S i}$ for a dilute Ni-Si alloy as a function of temperature. The superscript " 0 " in the Onsager coefficients corresponds to a strain-free system, whereas the superscripts " 11 " and " 33 " correspond to coefficients parallel and normal to the applied tensile strain, i.e. $\varepsilon_{33}=-2 \varepsilon_{11}=-2 \varepsilon_{22}=0.01$. The data points are direct measurements from atomistic KMC simulations for the strain-free case. Note the excellent agreement between the SCMF predictions and the KMC results for that case, and the direction-dependent effect of strain on the transition temperature from solute-drag $\left(L_{S i v} / L_{S i S i}>0\right)$ to IKE mediated diffusion of $\mathrm{Si}$ atoms. From Garnier et al. [58,59].

Vacancy-mediated diffusion in an infinitely dilute alloy on an fcc lattice involves for instance only 5 jump frequencies when the solute-vacancy interaction is limited to first nearest neighbors, as seen in Fig. 3.1. Morgan and collaborators [76-80] have employed this approach to model RIS in dilute Ni-Fe and Ni-Cr alloys, used as model systems to understand RIS in austenitic stainless steels. They calculated separately the contributions of RIS due to vacancy and interstitial fluxes as a function of temperature. This is important as earlier, phenomenological models by Perks and Murphy [81], Allen, Was et al. [82,83], and Grandjean et al. [65], concluded that existing data of irradiation-induced $\mathrm{Cr}$ depletion at grain boundaries could be reproduced by using parameters resulting in preferential vacancy-Cr exchanges, with no 
segregation contribution from interstitial fluxes. Some of these authors pointed out however that alternative parameterizations, where interstitial fluxes would contribute to RIS, could not be excluded. Barnard et al. [78] showed that in Ni interstitials would preferentially drag $\mathrm{Cr}$ to the grain boundaries, so that the net $\mathrm{Cr}$ depletion observed experimentally could in fact result from a competition between vacancy-driven $\mathrm{Cr}$ depletion and interstitial-driven $\mathrm{Cr}$ enrichment. Using ab initio molecular dyanmics (AIMD), Barnard and Morgan [79] proposed that the moderate Crdepletion reported experimentally resulted from a partial compensation of $\mathrm{Cr}$ depletion through vacancy diffusion and $\mathrm{Cr}$ enrichment through dumbbell interstitial migration, leading to much better agreement with experimental results, as illustrated in Fig. 4.6. A complete understanding of $\mathrm{Cr}$ segregation in austenitic steels remains an active research topic because the segregation effects are very subtle, and predictions could well be affected by small errors in DFT calculations, as well as by approximations regarding concentration effects, solute trapping, and defect clustering.

Other examples of the critical role played by first principles calculations can be found in results already discussed in this section. For instance, the calculation of the segregation or depletion tendencies of solute species in BCC iron, see Fig. 4.4, require the calculation of the relevant atomic jump frequencies for each solute element $\mathrm{X}(\mathrm{X}=\mathrm{Cr}, \mathrm{Cu}, \mathrm{Mn}, \mathrm{Ni}, \mathrm{P}, \mathrm{Si})$ in $\mathrm{Fe}$. Similarly, the SCMF predictions of the effect of strain on Onsager coefficients in dilute Ni-Si require the calculations of all relevant jump frequencies (up to 44 frequencies in the case of an applied shear strain).

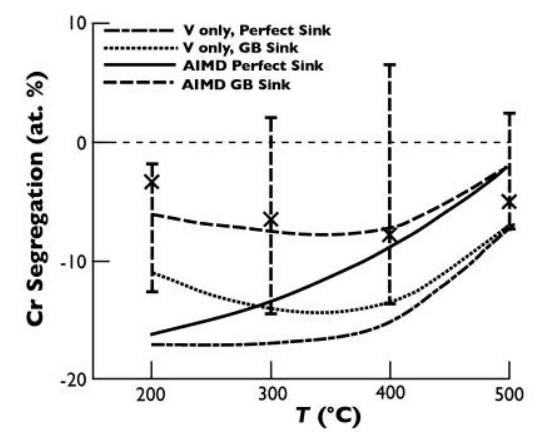

Figure 4.6. Radiation-induced segregation at grain boundary in Ni-Cr predicted by continuun models including contributions from vacancies only, for two different boundary conditions, and from ab initio molecular dynamics (AIMD) simulations, which include contributions from both vacancies and interstitials, compared to experimental results. Adapted from Barnard and Morgan [79]. 


\section{Experimental Observation of RIS}

RIS is most frequently found at assorted sinks in the microstucture, many of which preexist irradiation (e.g. grain boundaries and dislocations), and many of which are produced by irradiation itself (e.g. dislocation loops and voids). It is perhaps less well appreciated that RIS can also arise as a consequence of different kinds of self-organization. In the following sections we consider these various modes of RIS.

\subsection{Self-Organization from RIS}

The formation of phases that evidently nucleate homogenously under irradiation, yet clearly involve RIS, is relatively rare. A few examples are presented here. The first one is the observation of homogeneous precipitation of $\mathrm{Ni}_{3} \mathrm{Si}$ in a $\mathrm{Ni}-8$ at.\% $\mathrm{Si}$ alloy [13]. The microstructure of this alloy is shown in Fig. 5.1. Lam et al. [84] successfully explained this occurrence using a rate-theory model which predicted that the large flux of Si solute atoms into the mid-range region of the proton-irradiated specimens would enable the Si concentration to exceed the solubility limit at $600{ }^{\circ} \mathrm{C}$.

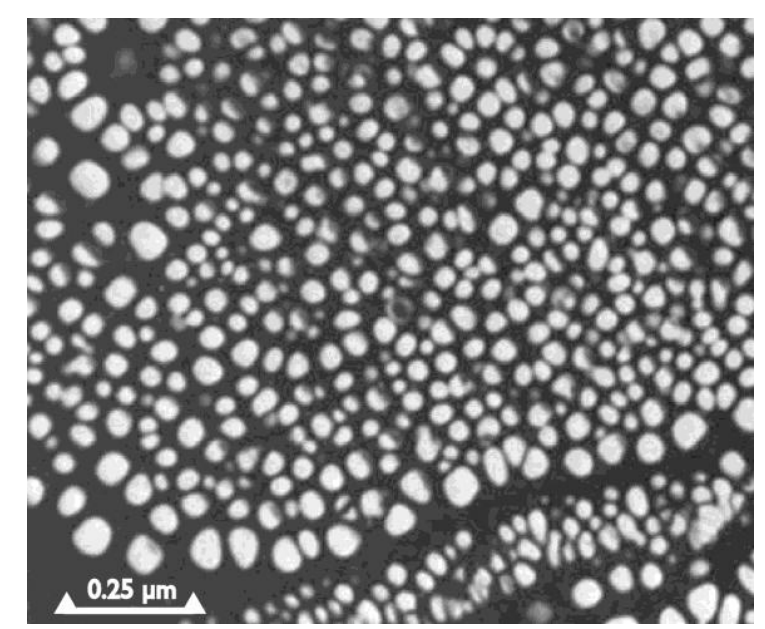

Figure 5.1. Radiation induced homogeneous precipitation of $\mathrm{Ni}_{3} \mathrm{Si}$ in a Ni-8 at.\% $\mathrm{Si}$ alloy irradiated at $600{ }^{\circ} \mathrm{C}$ by $400 \mathrm{keV}$ protons to a dose of $0.25 \mathrm{dpa}$ [13].

The explanation for homogeneous precipitation of $\mathrm{Ni}_{3} \mathrm{Si}$ in the experiments of Janghorban and Ardell [13] cannot possibly explain the more recent observations of the clustering of different kinds of precipitates in neutron-irradiated steels. Experimental observations of RIS at the smallest length scales have been aided immeasurably by the advent of 
atom-probe tomography (APT). The first reported observations of solute segregation using APT were those of Miller [85,86], who reported the segregation of $\mathrm{P}$ to dislocations in an assortment of steel specimens, and also observed $\mathrm{P}$ and/or $\mathrm{Cu}$-rich clusters that were evidently not associated with defects [85]. Though the alloys investigated by Miller were irradiated, not enough work was done to affirm that the segregation was radiation induced, though it probably was. Etienne et al. [87] investigated the redistribution of solute in $316 \mathrm{SS}$ in an alloy that was neutron irradiated to $12 \mathrm{dpa}$ at $633 \mathrm{~K}$ after having been in service for 17 years. Dislocation loops and small voids were seen using TEM, but Etienne et al. also observed a high number density (6 $\times 10^{23} \mathrm{~m}^{-3}$ ) of clusters $\sim 6 \times 8 \mathrm{~nm}$ in size enriched in $\mathrm{Ni}$ and $\mathrm{Si}$ but depleted in $\mathrm{Cr}$ and $\mathrm{Fe}$. Their results are reproduced in Fig. 5.2. Segregation of Mo and P at the interfaces of these clusters was also observed, and features described as atmospheres of Si were also reported. Etienne et al. [87] suggested that the Ni-Si-enriched clusters were associated with dislocation loops, but the association is tenuous and we would have no qualms about categorizing these findings as products of self-organization. Radiguet et al. [88] investigated the radiation-induced microstructures of nanocrystalline 316 SS prepared by high-pressure torsion. Using APT and TEM, they observed the RIS of Si and Ni at GBs, but did not observe Si-rich clusters in the grain interiors. It is possible that RIS to GBs eliminated the cluster formation seen by Etienne et al., but the differences reported in the 2 sets of experiments were not discussed, even though several researchers were involved in both sets of experiments.

Toyama et al. [89] reported the presence of uniformly spatially distributed Si-rich clusters in neutron-irradiated Type 304 stainless steel. An example of their results is shown in Fig. 5.3. Not only are the clusters enriched in $\mathrm{Si}$, they are also enriched in $\mathrm{Mn}, \mathrm{P}$ and $\mathrm{Ni}$, and somewhat depleted in Fe. Toyama et al. also observed dislocation loops in their specimens, but pointed out that the number density of clusters exceeded the number density of dislocation loops by nearly an order of magnitude $\left(4.2 \pm 2.1 \mathrm{cf} .0 .61 \pm 0.13 \times 10^{23} \mathrm{~m}^{-3}\right)$. The clusters were also resistant to annealing at $400{ }^{\circ} \mathrm{C}$, but coarsened on annealing at $500{ }^{\circ} \mathrm{C}$. These findings suggest that the clusters are possibly stable precipitates, the appearance of which is promoted by enhanced diffusion at $300{ }^{\circ} \mathrm{C}$. The concentrations of Si, Mn and P in the alloy were 1.5, 0.97 and 0.02 at. $\%$, respectively. The equilibrium solubilities these elements in 304 SS are unknown, so it is conceivable that enhanced diffusion provides an explanation, especially since the clusters are stable on annealing at higher temperatures. Until the solubility limits of these elements have been 
measured, the mechanism of their formation cannot be identified with certainty.

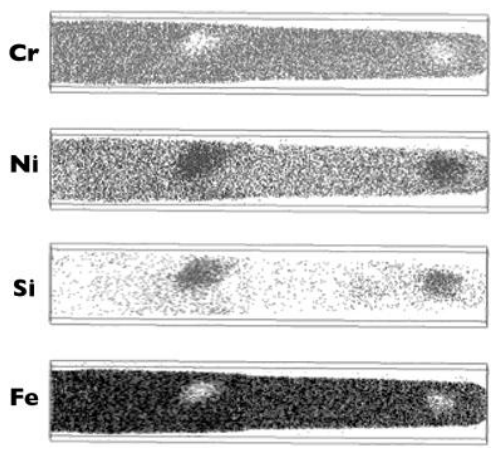

Figure 5.2. APT images of solute redistribution observed by Etienne et al. [87] in a Type 316 austenitic steel neutron irradiated at $633 \mathrm{~K}$ to a dose of $\sim 12 \mathrm{dpa}$. The length of each image (left to right) is $\sim 66 \mathrm{~nm}$ and the volume of the tip is slightly less than $6500 \mathrm{~nm}^{3}$. Si- and Ni-rich clusters are visible, while $\mathrm{Cr}$ and $\mathrm{Fe}$ are depleted in the same regions.
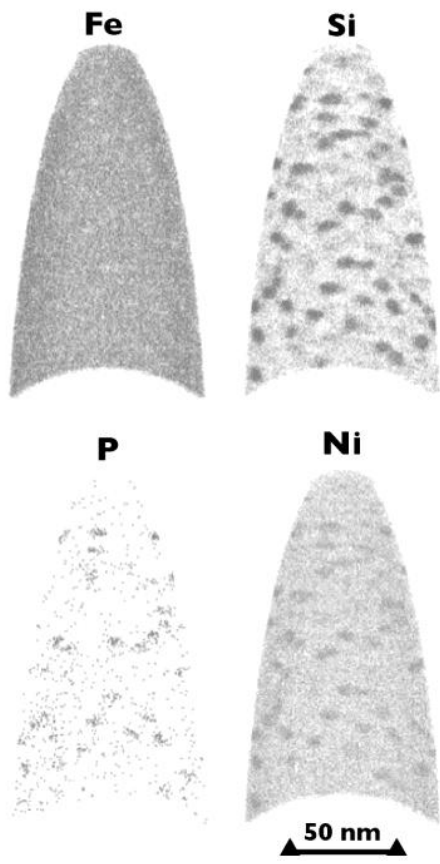
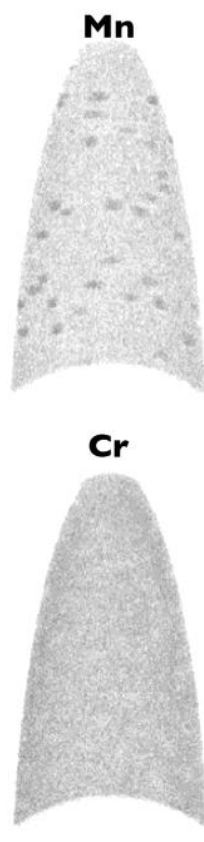

Figure 5.3. Elemental maps of solute atoms reported, using APT, by Toyama et al. [89] in a Type 304 austenitic stainless steel neutron-irradiated to a dose of $24 \mathrm{dpa}$ at $300{ }^{\circ} \mathrm{C}$. The volume of each tip is $\sim 2.56 \times 10^{5} \mathrm{~nm}^{3}$. Enrichment of $\mathrm{Si}, \mathrm{Mn}, \mathrm{P}$ and $\mathrm{Ni}$ is plainly visible. Depletion of $\mathrm{Fe}$ and $\mathrm{Cr}$ is observed, but is not quite as apparent.

Clustering during irradiation has also been observed by APT in neutron-irradiated ferritic or ferritic/martensitic (F/M) steels by Kuksenko et al. [90], as shown in Fig. 5 4. They found two kinds of clusters, Ni-Cr-Si-P rich with approximate compositions of 1.2, 24.2, 7.9 and 3.3 at.\%, 
respectively, and $\mathrm{Cr}$-rich clusters containing about 58.5 at. \% $\mathrm{Cr}$ and 0.65 at. $5 \mathrm{Si}$. They attributed the formation of Cr-rich clusters to irradiation-enhanced precipitation, since the alloy contained almost 11 at. \% Cr, but the formation of the Ni-Cr-Si-P clusters was attributed to RIS. In support of this conjecture Kuksenko et al. noted that they could not find a correlation between the clusters and point defect sinks such as dislocation loops.

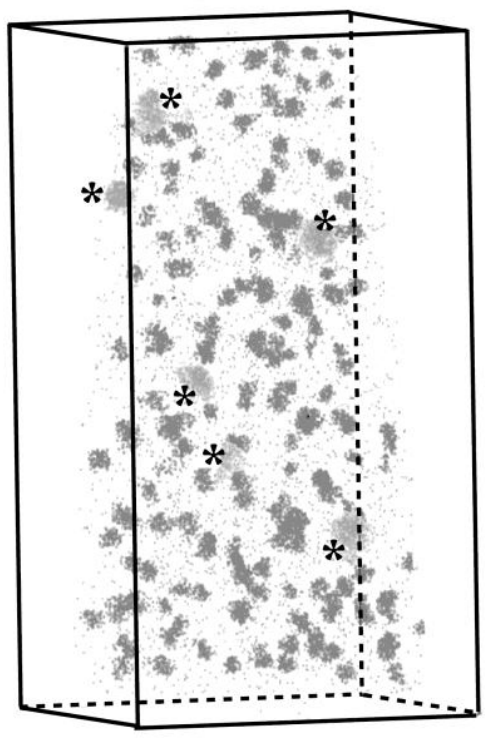

Figure 5.4. Radiation induced clusters observed by Kuksenko et al. [90] in a ferritic Fe-12 at. $\% \mathrm{Cr}$ steel after neutron irradiation at $300{ }^{\circ} \mathrm{C}$ to a dose of $0.6 \mathrm{dpa}$. Ni-Si-P clusters, 6 in all, are marked by asterisks, while $\mathrm{Cr}$-enriched clusters are the darker ones. The dimensions of the rectangle are $30 \times 30 \times 55 \mathrm{~nm}$.

Observations similar to those shown in Figs. 5.1 to 5.4 have been reported by other investigators in ferritic and austenitic steels. Meslin et al. [91] observed Mn-rich clusters in an ion-irradiated ferritic steel containing only 1 at. \% Mn. This alloy is undersaturated at the irradiation temperature of $400{ }^{\circ} \mathrm{C}$. The Mn-rich clusters were inhomogeneously distributed throughout the alloy, and while the authors suggested that the clusters formed as a consequence of RIS to a planar feature, that feature remains unidentified. Jiao and Was [92] observed a variety of clusters of different ehemistries by APT in a proton-irradiated F/M steel designated HCM12A. $\mathrm{Cu}$ and $\mathrm{Cr}$-rich precipitates were found and attributed to irradiation-enhanced diffusion, but there was no correlation reported between small $\mathrm{Ni} / \mathrm{Si} / \mathrm{Mn}$-rich precipitates and defects that could be positively identified as dislocation loops, so we surmise that these precipitates may have been radiation-induced by self-organization.

From a modeling perspective, homogeneous radiation-induced segregation and 
precipitation can be rationalized using the model developed by Cauvin and Martin [93-96]. This model captures the evolution of vacancies, interstitials, and solute atoms in a dilute alloy under irradiation. Under suitable thermodynamic and kinetic coupling between point defects and solute atoms, an undersaturated solid solution which is initially in thermodynamic equilibrium can become unstable under irradiation for highly specialized combinations of interactions and complexes among point-defects and solute atoms. The recombination of such complexes stimulates the spontaneous growth of local solute-rich zones, which can then evolve into soluterich precipitates. The original theory of Cauvin and Martin [93] was conceived to explain their observations of homogeneous precipitation of $\beta$-phase (essentially pure $\mathrm{Zn}$ ) particles in undersaturated binary Al-Zn alloys, which were High-Voltage Electron-Microscope (HVEM)irradiated at temperatures in excess of the solubility limit. An example of the microstructure is shown in Fig. 5.5

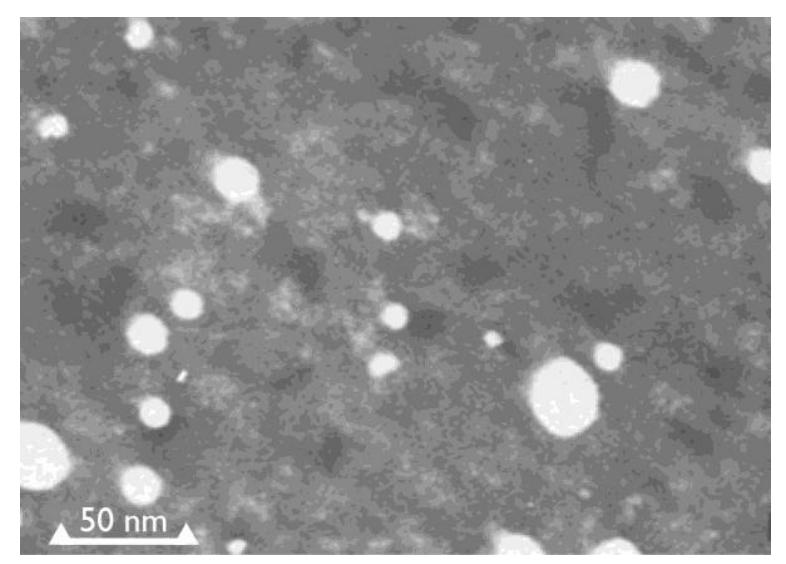

Figure 5.5. Dark-field images of $\beta$-phase $(\mathrm{Zn})$ particles in an undersaturated electron-irradiated Al-1.9at.\% Zn alloy electron-irradiated at $185^{\circ} \mathrm{C}$ at $9.1 \times 10^{-3} \mathrm{dpa} / \mathrm{s}$ to a dose of $45 \mathrm{dpa}$. From Cauvin and Martin [93].

A detailed analysis of the linear stability of candidate solid solutions under irradiation has been carried out by Martin [97], providing the general conditions for homogeneous RIS and RIP. A simpler requirement, presented by Cauvin and Martin [93], is that the solute should bind with either vacancies or interstitials, and that the solute flux should couple to point defect fluxes so as to promote the amplification of local solute composition fluctuations. Badillo et al. [98] used phase field modeling to explore this scenario. They considered an undersaturated $\mathrm{A}_{8} \mathrm{~B}_{92}$ alloy, where vacancies bind preferentially to solute atoms (component A), and where interstitials exclusively transport A atoms. As predicted by Cauvin and Martin for such a system, 
composition fluctuations grow after long enough irradiation dose, resulting in the formation of solute-rich precipitates, see Fig. 5.6. The physical origin of this instability is that regions with a slight solute-atom enrichment attract more vacancies, resulting in a higher local recombination rate, setting up in turn a net flux of point defects to these regions. In this case, the interstitial flux transports A atoms, and, after a recombination event, an A solute atom is left on a substitutional site in the region initially already enriched in solute, thus amplifying the growth of these composition fluctuations.

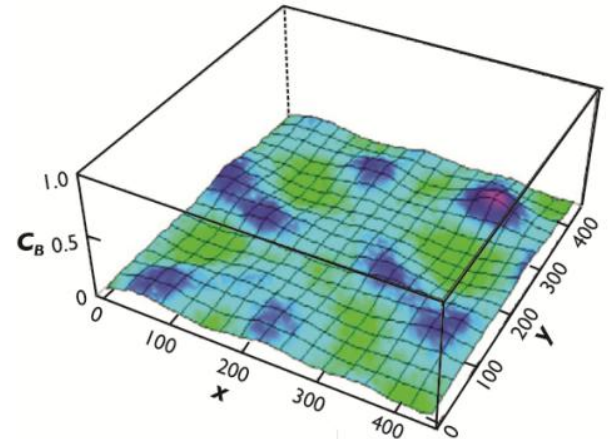

(a)

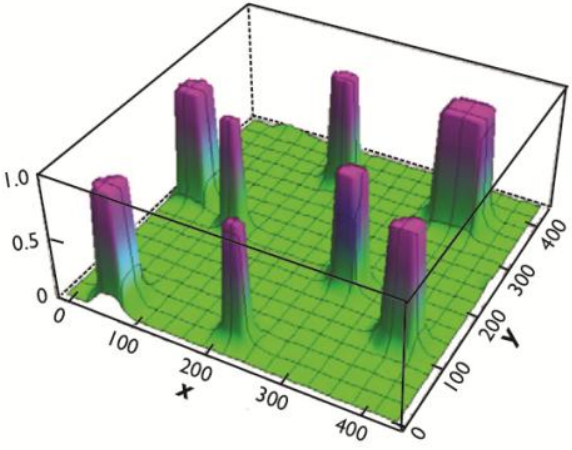

(b)

Figure 5.6. Irradiation-induced homogeneous precipitation in a two-dimensional undersaturated $\mathrm{A}_{8} \mathrm{~B}_{92}$ alloy with a positive heat of mixing. The local solute composition field is displayed as a function of spatial coordinates $\mathrm{x}$ and $\mathrm{y}$, in lattice cell units. The displacement rate is $2 \times 10^{-7}$ $\mathrm{dpa} / \mathrm{s}$; composition fluctuations developed after $11 \mathrm{dpa}(\mathrm{a})$, and resulted in the formation of preciptiates after 40 dpa (b). Computational modeling results of Badillo et al. [98].

\subsection{RIS at Dislocations}

The initial observations of RIS at dislocations was reported by Barbu and Ardell [10], who conjectured correctly that RIS was responsible for the precipitation of $\mathrm{Ni}_{3} \mathrm{Si}$ at faulted dislocation loops in an undersaturated binary Ni-Si alloy (see Fig. 2.1). RIS at faulted dislocation loops in neutron-irradiated austenitic stainless steel was first reported by Kenik [99], who used AEM to observe the segregation of $\mathrm{Cr}$ at edge-on loops. There have since been numerous observations of precipitation at dislocation loops in irradiated fcc alloy solid solutions. RIS to dislocation loops in bcc alloys was not observed until the work of Wakai et al. [31]. They reported the appearance of the $\alpha^{\prime}$ phase in binary Fe-Cr alloys HVEM-irradiated from 300 to 700 ${ }^{\circ} \mathrm{C}$ by $1 \mathrm{MeV}$ electrons, and attributed the formation of the $\alpha^{\prime}$ phase to RIS of $\mathrm{Cr}$.

In a subsequent investigation of RIS in Type 316 austenitic stainless steel, Etienne et al. [100] found different intragranular features, including perhaps the first observation using APT of 
Si-enriched torus-shaped clusters (see Fig. 5.7); these could only have formed by RIS to dislocation loops. Interestingly, the enrichment of $\mathrm{Si}$ at different kinds of sinks was different, being lowest at GBs and highest at "rounded" clusters. The magnitude of RIS measured by Etienne et al. was higher than what was previously reported in the literature, prompting the authors to suggest that techniques other than APT might underestimate the peak values of RIS.

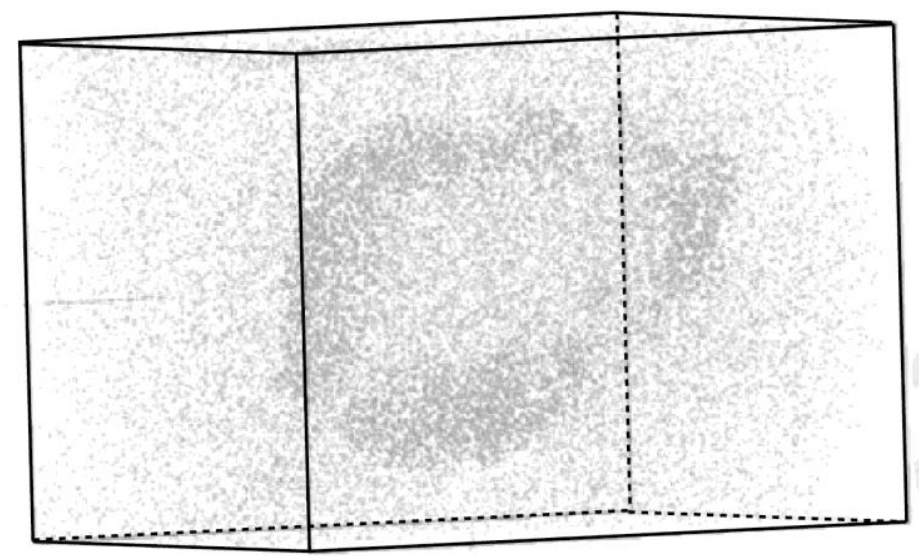

Figure 5.7. A Si loop shaped cluster observed by Etienne et al. in a Type 316 austenitic steel irradiated at $350{ }^{\circ} \mathrm{C}$ by $\mathrm{Fe}^{5+}$ ions to a dose of $\sim 10 \mathrm{dpa}$. The height of the bounding box is $34 \mathrm{~nm}$.

Additional work by Etienne et al. [101] on $\mathrm{Fe}^{+}$-ion irradiated 316 SS clearly showed the formation of Frank loops and solute clusters enriched in Si and, in some cases, also enriched in $\mathrm{Ni}$ and depleted in $\mathrm{Cr}$. Irradiation at $350{ }^{\circ} \mathrm{C}$ to doses between 0.5 and 5 dpa established a convincing correlation between the number densities of dislocation loops and solute clusters. This result, which is completely at variance with the findings of Toyama et al. [89] in their neutron-irradiated Type 304 stainless steel (Fig. 5.3), suggests that solute clusters nucleate heterogeneously on dislocation loops and that $\mathrm{Si}$ atoms are dragged to sinks by an interstitial mechanism. The results obtained from specimens irradiated at 300 and $400{ }^{\circ} \mathrm{C}$ showed that the extent of solute enrichment increased with increasing irradiation temperature

RIS to dislocation loops in bcc alloys has been observed by several investigators [31,32,102]. Wakai et al. [31] interpreted contrast in TEM images of an electron-irradiated binary $\mathrm{Fe}-\mathrm{Cr}$ alloy as evidence the segregation of $\mathrm{Cr}$ to dislocation loops, forming the $\alpha^{\prime}$ phase. Bhattacharya et al. [32] thoroughly investigated the irradiation-induced microstructures of binary $\mathrm{Fe}-\mathrm{Cr}$ alloys using dual-beam $\left(\mathrm{He}^{+}\right.$and $\left.\mathrm{Fe}^{+}\right)$irradiations to a range of doses at $400{ }^{\circ} \mathrm{C}$. An example of the contrast observed at large planar loops is shown in Fig. 5.8, where the segregation is manifested as displacement fringe contrast. The segregation of $\mathrm{Cr}$ to the 
dislocation loops was measured using both Scanning-Transmission-Electron-Microscopy (STEM) and APT, and the Cr concentration on the loops was found to be smaller than the concentration of the $\alpha^{\prime}$ phase at the irradiation temperature, which suggests that the displacement fringe contrast seen in Fig. 5.8 is not a consequence of RIP of the $\alpha^{\prime}$ phase. $\mathrm{Cr}$ is oversized in bcc $\alpha-\mathrm{Fe}$ (see the compilation of data by Ghosh and Olson [27]), and the mechanism of segregation to dislocation loops has not yet been identified, but might involve the participation of interstitially dissolved carbon atoms.

As is the case for fcc alloys, $\mathrm{Si}$ is undersized in $\alpha-\mathrm{Fe}$ [27], so perhaps it is not surprising that $\mathrm{Si}$ segregates to dislocation loops in irradiated ferritic and F/M steels. Jiao and Was [102] report the enrichment of $\mathrm{Si}$, as well as $\mathrm{Ni}, \mathrm{Mn}$ and $\mathrm{Cu}$, on dislocation loops in the $\mathrm{F} / \mathrm{M}$ steel HCM12A. An example of the RIS of Si at a dislocation loop is shown in Fig. 5.9. Interestingly, Jiao and Was also find enrichment of $\mathrm{Cr}$ in the interior of dislocation loops of comparable size to that in Fig. 5.9, which is consistent with the results of Wakai et al. [31] and Bhattacharya et al. [32].

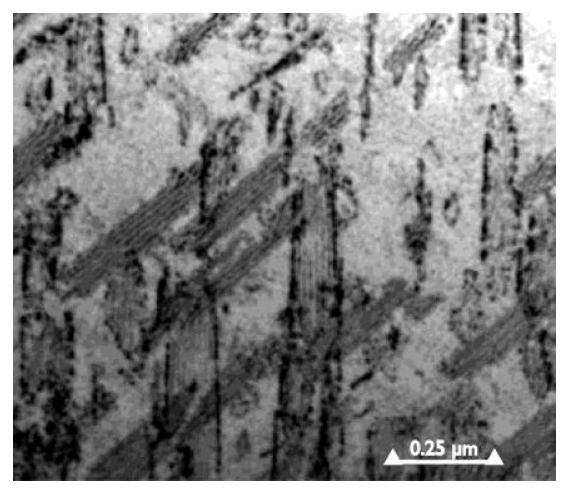

Figure 5.8. Planar dislocation loops observed by Bhattacharya et al. [32] in a Fe-5.8 at.\% $\mathrm{Cr}$ alloy irradiated by $2 \mathrm{MeV} \mathrm{Fe}^{2+}$ ions to a dose of $45 \mathrm{dpa}$ at $500{ }^{\circ} \mathrm{C}$. The weak contrast in the interior of the elongated loops is interpreted as displacement fringe contrast brought about by the segregation of oversized $\mathrm{Cr}$ atoms. 


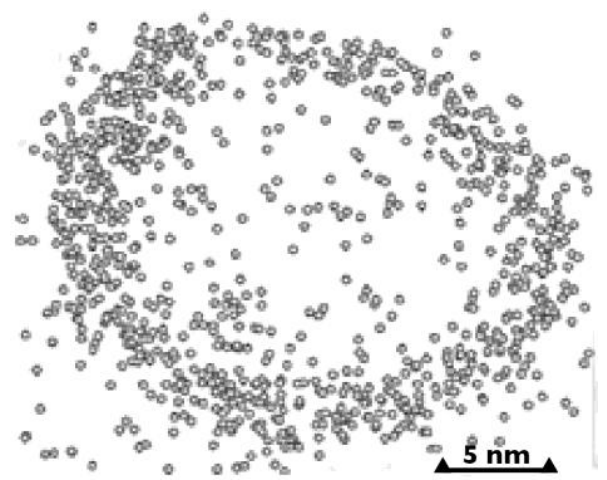

Figure 5.9. RIS of Si to a dislocation loop observed by Jiao and Was [102] in the ferritic steel $\mathrm{HCM} 12 \mathrm{~A}$ irradiated by $2 \mathrm{MeV}$ protons at $400{ }^{\circ} \mathrm{C}$ to a dose of $7 \mathrm{dpa}$.

Following the early work of Etienne et al. [87,100,101] and Radiguet et al. [88], the subsequent research using APT to examine radiation damage has been highly impactful. Several studies $[103,104]$ have directly revealed RIS at dislocations and dislocation arrays other than dislocation loops. Williams et al. [103] found Mn segregation to what appears to be a dislocation network, Fig. 5.10, in an oxide-dispersion-strengthened (ODS) ferritic steel. Kuksenko et al. $[104,105]$ observed segregation to dislocations in low-angle grain boundaries in model ferritic steels ( 9 and 12 at. $\% \mathrm{Cr}$ ) irradiated to $0.6 \mathrm{dpa}$ by neutrons at $300{ }^{\circ} \mathrm{C}$. Examples of Si segregation to dislocations at twist and tilt boundaries, respectively, are shown in Figs. 5.11 and 5.12; composition profiles normal to the dislocations in the tilt boundary are also shown in Fig. 5.12. It is evident in Fig. 5.12 that $\mathrm{Cr}$ and $\mathrm{P}$, as well as $\mathrm{Si}$, segregate to the edge dislocations in the lowangle tilt boundary.

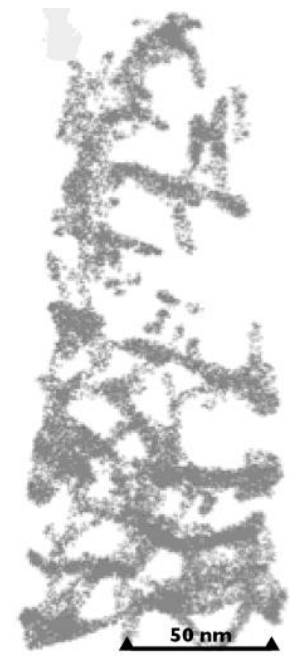

Figure 5.10. Segregation of Mn to a dislocation network in the ODS ferritic steel ODS Eurofer 97 irradiated by $\mathrm{Fe}^{2+}$ ions to a dose of $2 \mathrm{dpa}$ at $400{ }^{\circ} \mathrm{C}$; from Williams et al. [103]. 


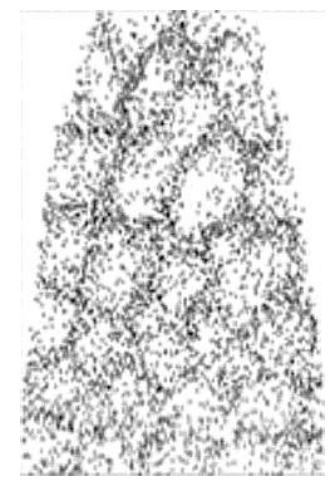

Figure 5.11. Segregation of $\mathrm{Si}$ at a hexagonal GB dislocation network in a Fe-12 at.\% $\mathrm{Cr}$ alloy neutron-irradiated at $300{ }^{\circ} \mathrm{C}$ to a dose of $0.6 \mathrm{dpa}$. The dimensions of the image are approximately $50 \times 70 \mathrm{~nm}$; from Kuksenko et al. [104].
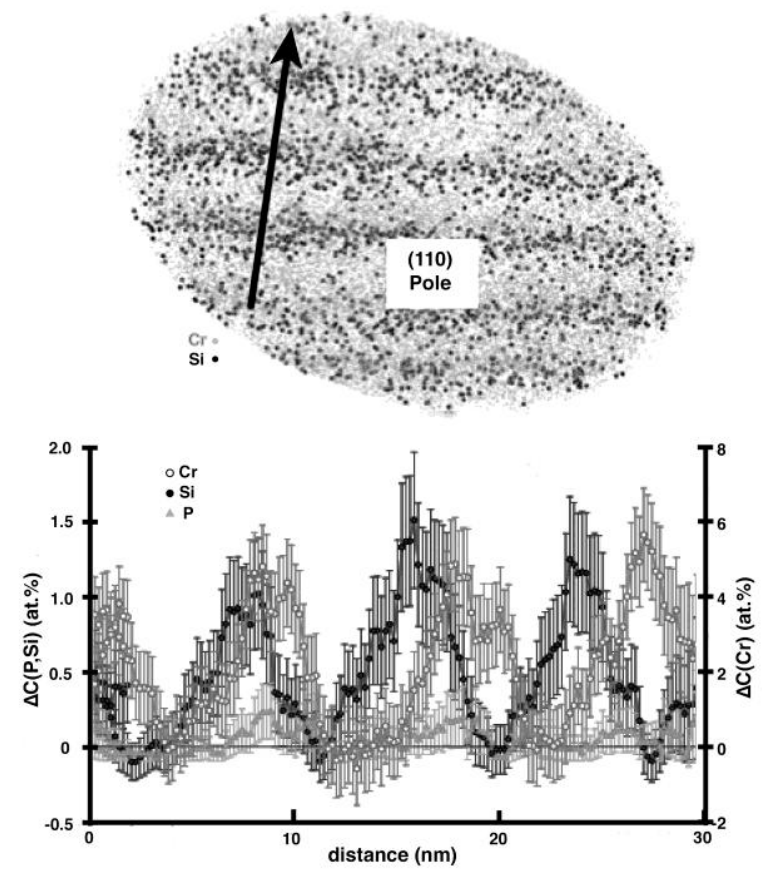

Figure 5.12. Elemental map showing the distributions of $\mathrm{Cr}$ and $\mathrm{Si}$ in a low-angle tilt boundary (upper image) in a ferritic Fe-12 at.\% Cr steel neutron irradiated at $300{ }^{\circ} \mathrm{C}$ to a dose of 0.6 dpa [105]. The lower image shows the measured variations in concentration of $\mathrm{Cr}, \mathrm{Si}$ and $\mathrm{P}$ along the line indicated by the arrow in the upper image. The authors attribute the displacements of the $\mathrm{Cr}$ and $\mathrm{Si}$ peaks to the size differences between the atoms, $\mathrm{Si}$ being undersized and $\mathrm{Cr}$ being oversized.

Jiao and Was [106] investigated RIS in 3 austenitic steels, including commercial purity 304 (their designation CP304). The irradiated microstructures were investigated using APT. Both substitutional and interstitial atoms were found to segregate to the loops, but in contradistinction to most measurements, the concentrations were observed to vary around the loop, as shown for $\mathrm{Ni}$ and Si atoms in Fig. 5.13. 


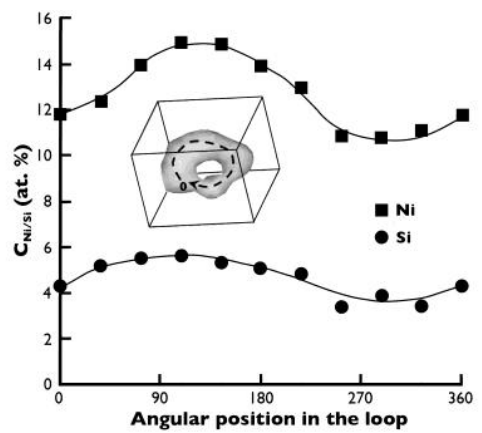

Figure 5.13. Variations in the composition of $\mathrm{Ni}$ and $\mathrm{Si}, C_{N i / S i}$, around a dislocation loop in a commercial purity Type 304 austenitic stainless steel irradiated by $2 \mathrm{MeV}$ protons to a dose of 5 dpa at $360{ }^{\circ} \mathrm{C}$ [106]. The diameter of the loop is 12 to $14 \mathrm{~nm}$ and the measured concentrations were taken in a clockwise direction around the loop (see inset).

RIS of Si to pure screw dislocations in a neutron-irradiated type 316 stainless steel has been reported by Hatakeyama et al. [107]. Measurements by APT of the concentration profile across the core of a screw dislocation are shown in Fig. 5.14. The attraction between undersized $\mathrm{Si}$ atoms and a screw dislocation, with no dilatational component, is indeed an interesting finding, since screw dislocations are unexpected dislocation sinks. At the same time, RIS to screw dislocations in the network in Fig. 5.11 is consistent with the observations of Hatakeyama et al. In other recent work Hatakeyama et al. [108] observed the segregation of $\mathrm{Si}, \mathrm{Cr}$ and $\mathrm{Ni}$ to stacking faults in the same neutron irradiated stainless steel. We regard screw dislocations and stacking faults as relatively weakly biased point-defect sinks, yet they are quite effective in serving as sinks for RIS. It would be quite interesting to model such effects and determine whether their strength is consistent with these experimental observations.

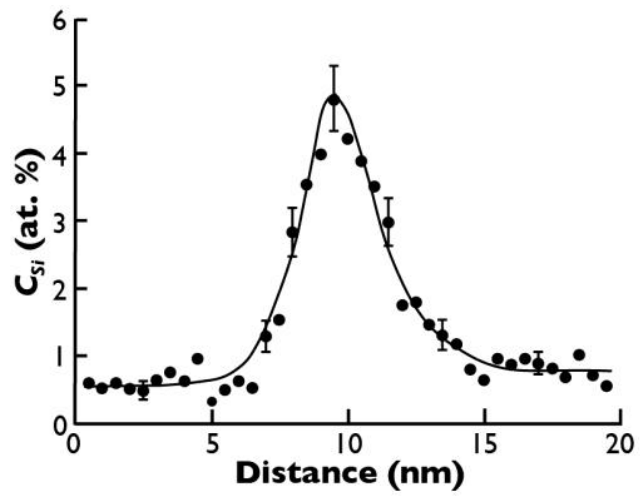

Figure 5.14. Concentration of $\mathrm{Si}, C_{S i}$, as a function of distance across a screw dislocation in a modified Type 316 austenitic stainless steel neutron-irradiated at $589{ }^{\circ} \mathrm{C}$ to a dose of $56 \mathrm{dpa}$. Hatekayama et al. [107] reported error bars for all their data, but only a few representative error bars are shown. 


\subsection{RIS at Grain Boundaries}

There have been numerous observations of RIS at grain boundaries in alloys. Many of the impactful papers in this field of research are cited in review articles by Ardell [1], Nastar and Soisson [2], Faulkner [109], and Wharry and Was [110]. Most of the research conducted within the past decade or so in this area of radiation effects has concentrated on RIS at grain boundaries in steels that are candidates for use in so-called Generation-IV nuclear reactors. The steels in question are either austenitic, ferritic or ferritic/martensitic. The remainder of this section will focus on grain boundaries in these kinds of alloys and the factors that affect RIS, the most important of which are initial GB solute concentration, GB structure and GB mobility. Other potentially important factors are the effect of oversize solute atoms and, at the other end of the size spectrum, the role of interstitial atoms, as well as dose, dose rate and bombarding species. In this section we review the existing literature on these topics and describe the extent to which they are understood mechanistically.

\subsubsection{Role of Initial GB Concentration}

Atoms in solid solution segregate to grain boundaries as components of the equilibrium microstructure. The literature on this topic is rich with examples. The reader is referred to several excellent review articles on the thermodynamic and structural aspects of equilibrium GB segregation and its manifestations, including TNES [111-114]. The extent of GB segregation is sometimes called the Gibbsian excess. We tend to think that the coverage of GBs by the segregation of solute atoms is uniform, which is the way the Gibbsian excess is treated thermodynamically, but the examination of GB by APT indicates that this is not the case, even for random high-angle GBs. From atomistic simulations of segregation at heterointerfaces, Demkowicz et al. [115] proposed that, at boundaries that can be described by a network of misfit dislocations, the solute segregation energy should in fact be considered as spatially heterogeneous. This is accord with the observations of Tomozawa et al. [116], who show that the segregation of solute atoms to GB is inhomogeneous, even in unirradiated specimens. Examples are shown in Fig. 5.15, taken from an examination of random GBs in an unirradiated type 316L stainless steel. A map of the Gibbsian excess of $\mathrm{Cr}$ in an unirradiated ferritic steel is seen in Fig. 5.16, taken from the work of Miller and Yao [114]. The importance of these findings is that 
segregation prior to irradiation impacts not only the concentrations of atoms that are enriched or depleted at GBs, but also their spatial distributions.

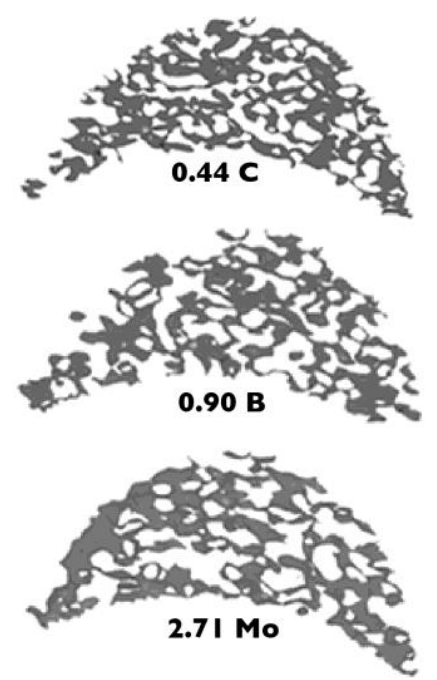

Figure 5.15. Elemental spatial distributions (dark patches) of C, B and Mo in the plane of a random grain boundary in an unirradiated Type 316 stainless steel [116]. The numbers below each image represent the concentration, in at. \%, of the element. Each image is approximately 82 $\mathrm{nm}$ in width.

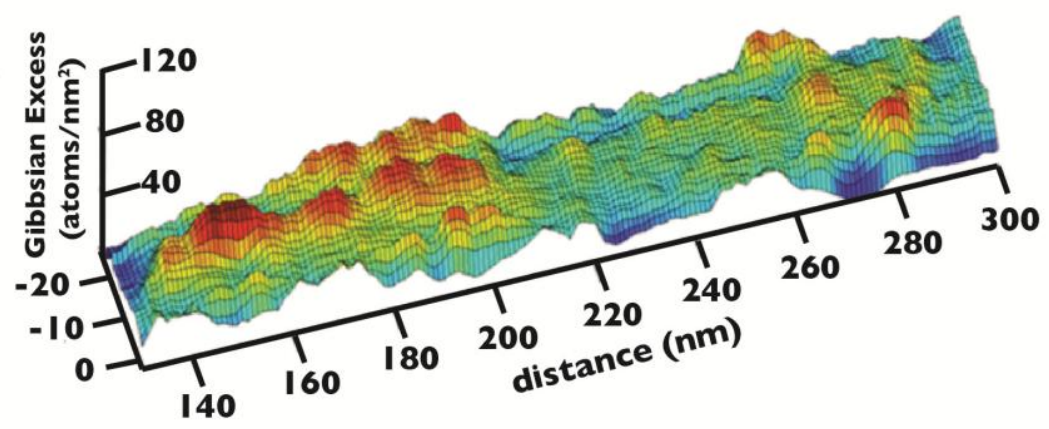

Figure 5.16. Gibbsian interfacial excess of $\mathrm{Cr}$ in a grain boundary in an unirradiated mechanically alloyed ferritic steel 14 YWT NFA, annealed for $24 \mathrm{~h}$ at $500{ }^{\circ} \mathrm{C}$ [114].

The results shown in Figs. 5.15 and 5.16 demonstrate convincingly that the solute enrichment produced by TNES is not uniform in the plane of the grain boundary. These findings would have been nearly impossible by early examinations by AEM, such as those of Goodwin et al. [36] shown in Fig. 2.4, which average the concentrations within the boundary through the thickness of the thin foil specimens. It is nevertheless instructive to learn what happens at GBs on irradiation of specimens in which TNES has occurred. An example from the work of Goodwin et al. is shown in Fig. 5.17. At an intermediate dose of 1 dpa the so-called "W-shaped 
profile" is observed, while at larger doses the profile becomes more comparable to those in steels in which TNES has not occurred, i.e. a monotonically decrease of the Cr concentration as the GB is approached. These findings of Goodwin et al. are not unique. Busby et al. [117] observed similar behavior in proton-irradiated type 304 and 316 stainless steels cooled from high temperatures by forced air convection, though the magnitude of TNES was not quite as large. The features seen in Fig. 5.17 are representative of TNES and RIS of various elements in austenitic and ferritic steels $[117,118]$ and will be discussed in more detail in Sec. 5.5.

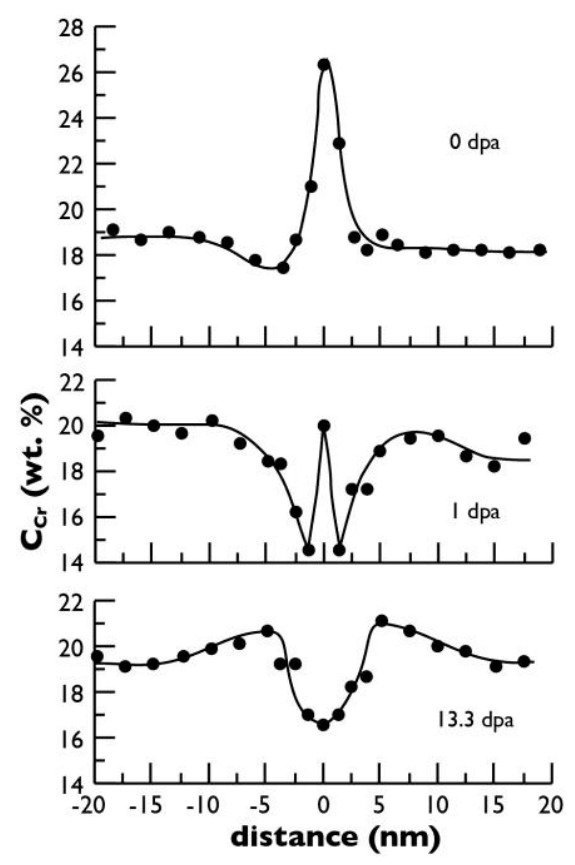

Figure 5.17. Data on the $\mathrm{Cr}$ concentration, $C_{C r}$, profiles in a moderately rapidly cooled Type 304 austenitic stainless steel, neutron-irradiated at $288{ }^{\circ} \mathrm{C}$ to the doses indicated. The uppermost plot illustrates TNES in an unirradiated specimen (see also Fig. 2.4). The W-shaped profile at intermediate doses is shown in the middle plot and normal RIS is observed at higher doses. Data of Goodwin et al. [36].

\subsubsection{RIS at General High-Angle Grain Boundaries}

RIS at grain boundaries in steels was first measured quantitatively using analytical electron microscopy (AEM) by Norris et al. [41]. They investigated radiation effects in an austenitic steel containing $\sim 20 \% \mathrm{Cr}, \sim 25 . \% \mathrm{Ni}$ and $\sim 0.8 \% \mathrm{Nb}$ (all wt. \%) irradiated by neutrons in an advanced gas-cooled reactor. They did not report any segregation in the unirradiated alloy and we assume that no evidence of TNES was found. Representative results on the segregation of $\mathrm{Ni}$ and Fe are shown in Fig. 2.6. As seen in Fig. 2.6 Ni, which is undersized, is enriched while 
Fe is depleted at GBs. At higher temperatures the concentration profiles are much broader, reflecting the increasingly important role of recombination as temperature increases. Kenik [99] observed profiles similar to those of Norris et al. in neutron-irradiated Type 304 SS of commercial purity, but also noted that segregation of Si and P was considerably lower in highpurity steel. It is often found that the concentration profiles produced by RIS at grain boundaries do not depend monotonically on distance from the boundary. This is evident for the profiles of $\mathrm{Fe}$ and $\mathrm{Ni}$ in Fig. 2.6, but the profile for $\mathrm{Cr}$ (not shown) decreases monotonically as the GB is approached [41]. This is very different from what is seen in Fig. 5.17 and could very well be a consequence of the absence of TNES in the alloy studied by Norris et al. [41].

An excellent recent example of RIS at GBs in Type 304 stainless steel is seen in the work of Toyama et al. [119]. Using laser-assisted APT, they reported the enrichment of Ni, Si, and P and the depletion of $\mathrm{Cr}$ and $\mathrm{Fe}$ at a grain boundary, the character of which was not specified. The APT images for C, Mn, Si, P, Ni and Cr are shown in Fig. 5.18. RIS of C and Mn was not observed. Concentration profiles through the GB are shown in Fig. 5.19. The full-width at halfmaximum of the RIS region was $\sim 3 \mathrm{~nm}$ for the concentration profile peaks of $\mathrm{Ni}$ and $\mathrm{Si}$. The atomic percentages of $\mathrm{Ni}, \mathrm{Si}$, and $\mathrm{Cr}$ at the $\mathrm{GB}$ were $\sim 19 \%, \sim 7 \%$, and $\sim 14 \%$, respectively, which is consistent with previously reported values for neutron-irradiated austenitic stainless steels (see Fig. 2.6 for Ni-enrichment and Fig. 5.17 for Cr depletion). A high number density of intragranular Ni-Si rich precipitates formed in the matrix. A precipitate-denuded zone with a width of $\sim 10 \mathrm{~nm}$ appeared on both sides of the GB.

Though it is not at all evident in Figs. 5.18 and 5.19, it is important to realize that the distributions of solute in the plane of the GB are not homogeneous, just as the solute that segregates to GBs in unirradiated specimens is not homogeneous. This is nicely illustrated by the results of Jiao and Was [106], who observed significant variations in the concentration of the segregated elements within the grain boundary plane in their proton-irradiated specimens of type 304 SS. Examples of the variations of $\mathrm{Cr}$ and $\mathrm{Si}$ within the GB plane are shown in Fig. 5.20. Jiao and Was report that the concentration of $\mathrm{Cr}$ varies from 7.8 to 19.1 at. \%, while that of Si varies from 3.5 to $9.1 \%$ in a steel with nominal concentrations of $19.2 \% \mathrm{Cr}$ and $2.05 \% \mathrm{Si}$. The depletion of $\mathrm{Cr}$ and enrichment of Si observed by Jiao and Was [106] is consistent with the data of Toyama et al. [119], but the variations of concentration of these species should come as no 
surprise when viewed in the light of the results of Tomozawa et al. [116] and Miller and Yao [114] on unirradiated steels (Figs. 5.15 and 5.16).
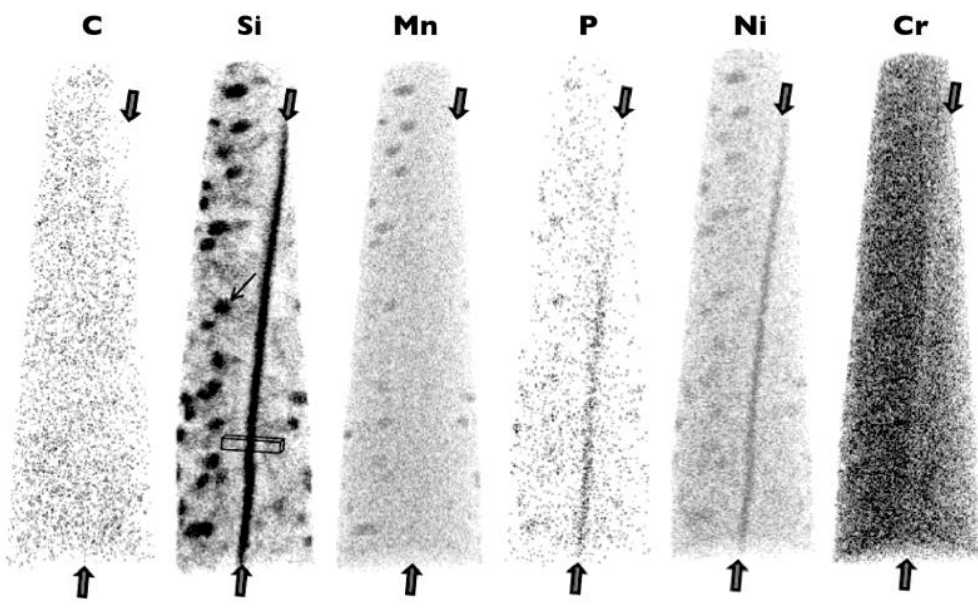

Figure 5.18. Concentration of the indicated elements at GBs in a tip of neutron irradiated Type 304 stainless steel, neutron-irradiated to a dose of $24 \mathrm{dpa}$ at $300{ }^{\circ} \mathrm{C}$; results of Toyama et al. [119]. The box inset in the image for Si represents the region used to trace the elemental concentrations across the GB (see Fig. 5.19); the box is $\sim 20 \mathrm{~nm}$ long.

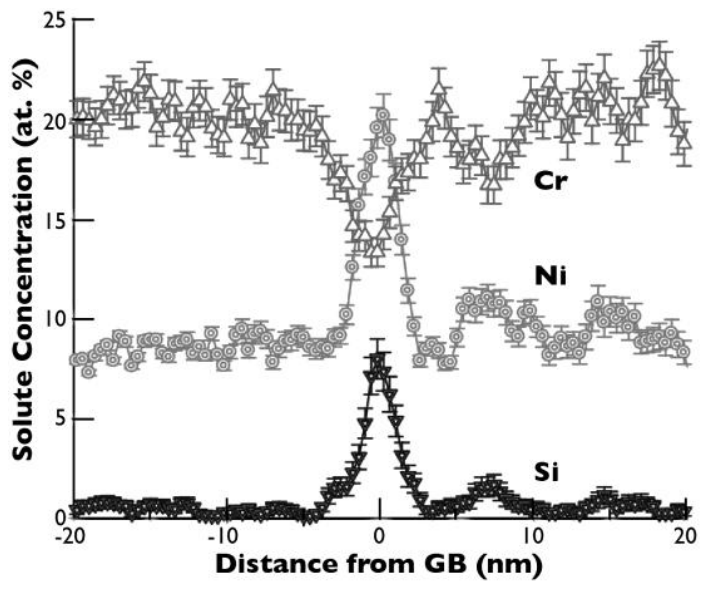

Fig. 5.19. Composition profiles of $\mathrm{Cr}, \mathrm{Ni}$ and $\mathrm{Si}$ normal to grain boundary planes in Type 304 stainless steel neutron irradiated at $300{ }^{\circ} \mathrm{C}$ to a dose of $24 \mathrm{dpa}$. Data of Toyama et al. [119]. 

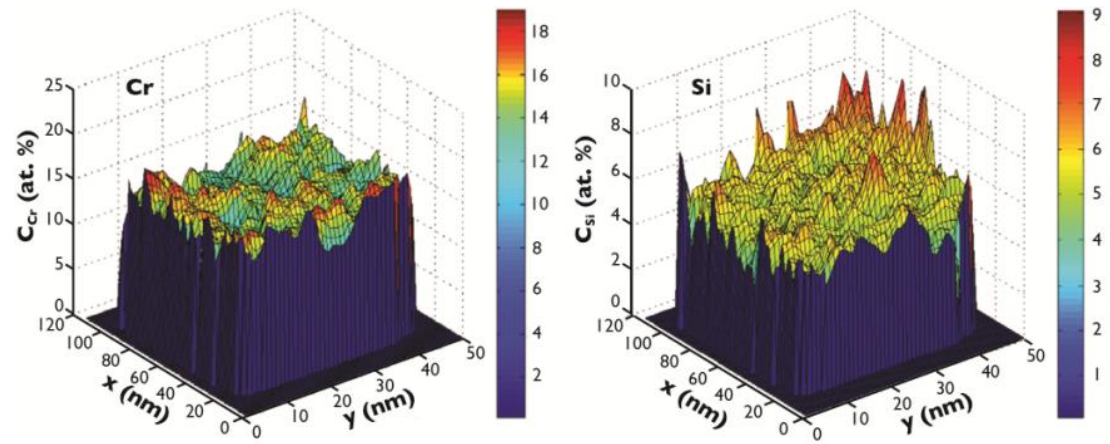

Fig. 5.20. Composition variations of $\mathrm{Cr}$ and $\mathrm{Si}, C_{C r}$ and $C_{S i}$ respectively, in the grain boundary planes in HP304 + Si following irradiation to $5 \mathrm{dpa}$ at $360{ }^{\circ} \mathrm{C}$. Distance coordinates in the plane of the grain boundary[119] are represented by x and y. Data of Jiao and Was [106].

Allen et al. [120] investigated the effect of displacement rate on radiation-induced segregation in samples of Types 304 and 316 stainless steel. They compared the microstructures in specimens irradiated at low dose rates in the EBR-II nuclear reactor with those in specimens proton-irradiated at much higher dose rates. Despite mitigating factors, the results suggest that decreasing the dose rate leads to greater radiation-induced segregation. Specifically, increasing the dose rate leads to lower depletion of $\mathrm{Cr}$, higher enrichment of $\mathrm{Si}$ and lower enrichment of $\mathrm{Ni}$ at point defect sinks. However, the absence of complete control over all the experimental variables (e.g. the nature of the knock-on damage produced by protons and neutrons) indicates that this conclusion should be regarded with some caution, though additional evidence supporting it has recently been presented by Allen et al. [7].

Ferritic and F/M steels generally have more complicated microstructures than austenitic steels, one of the main differences being that the structures of F/M interfaces can have features that do not exist in single-phase alloys. Nevertheless, the radiation-induced microstructures in ferritic and F/M steels share much in common with those observed in austenitic steels. These include the depletion of certain atomic species and the enrichment of others at GBs, the supression of RIS by the addition of oversized solute atoms, RIS at migrating boundaries, etc. A few examples follow.

Muroga et al. [121] have observed the depletion of $\mathrm{Cr}$ and enrichment of $\mathrm{Ni}$ at ferriteferrite grain boundaries in steel containing $10 \% \mathrm{Cr}$ and $5 \% \mathrm{Ni}$ (wt.); this result is shown in Fig. 5.21. In other respects $\mathrm{Cr}$ behaves quite differently in ferritic and F/M steels from its behavior in austenitic steels in that GBs are sometimes enriched rather than depleted in Cr. For example, Lu et al. [122] observed both enrichment and depletion in ferritic steels irradiated by a variety of different species (electrons, protons, heavy ions). Even though $\mathrm{Cr}$ is clearly an oversized solute 
atom in ferritic and F/M steels [27], Lu et al. speculate that under some conditions in certain alloys $\mathrm{Cr}$ might be considered undersized and behave accordingly. Wharry and Was [123] find that $\mathrm{Cr}$ is enriched at GBs in the F/M steel T91 under most irradiation conditions at temperatures below $700{ }^{\circ} \mathrm{C}$, but is depleted at $700{ }^{\circ} \mathrm{C}$, all other irradiation conditions being equal. They modeled this behavior using a modification of their own inverse Kirkendall mechanism [124], but acknowledge that their model does not explain all aspects of the observed behavior. Marquis et al. [125] and $\mathrm{Hu}$ et al. [126] have reported instances of $\mathrm{Cr}$ being both enriched and depleted at GBs in an oxide-dispersion strengthened ferritic steel that was ion-implanted at $500{ }^{\circ} \mathrm{C}$. Clearly, there is no consistency with regard to the role played by irradiation temperature as far as the segregation or depletion of $\mathrm{Cr}$ is concerned. In this context we note that Wei et al. [127] found neither $\mathrm{Cr}$ enrichment nor depletion in T91 ferritic steel irradiated at $450{ }^{\circ} \mathrm{C}$ to $150 \mathrm{dpa}$, so it appears that all traces of RIS are eliminated after irradiation to ultra-high doses, at least in this ferritic steel.

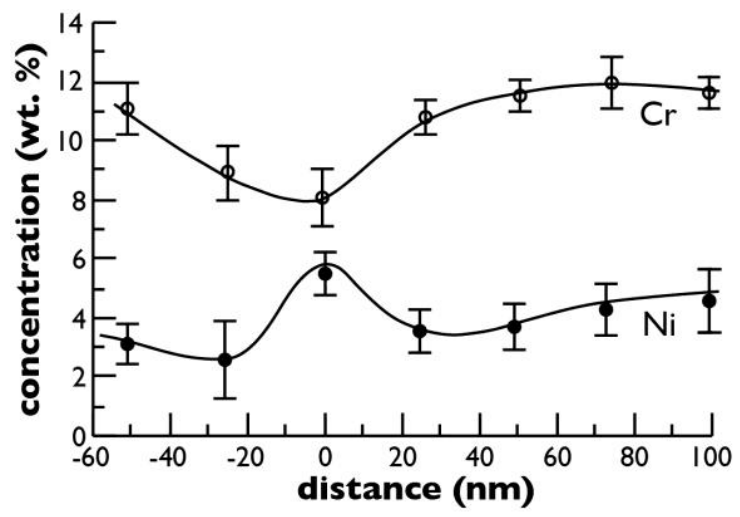

Figure 5.21. Concentration profiles of $\mathrm{Ni}$ and $\mathrm{Cr}$ at grain boundaries in a ferritic steel irradiated at $500{ }^{\circ} \mathrm{C}$ to a dose of $3 \mathrm{dpa}$ using $1 \mathrm{MeV}$ electrons. Data of Muroga et al. [121].

\subsubsection{RIS at Moving Grain Boundaries}

Grain boundaries in alloys can migrate under irradiation conditions. In such cases the segregation profiles are not symmetric, with the deviations from symmetry often quite large. An example taken from the work of Norris et al. [41] is shown in Fig. 5.22. RIS associated with radiation-induced GB migration has also been observed directly by Sakaguchi et al. [128] in an austenitic stainless steel; their results are shown in Fig. 5.23. Sakaguchi et al. developed a model based on the WOL theory that incorporates the velocity of the GB via a parameter $\alpha_{v, i}$, called the rearrangement factor, which depends on the difference between the radiation-induced and 
equilibrium vacancy concentrations. For $\alpha_{v, i}=0$ RIS is predicted to occur without GB migration, but for values of $\alpha_{v, i}$ as small as $10^{-8}$ their model predicts measurable migration. Comparisons between the model of Sakaguchi et al. and experimental data are shown in Fig. 5.23 where it is evident that the agreement, while not perfect, is quite good.

Sakaguchi et al. [128] note that the physical significance of the parameter $\alpha_{v, I}$ is not well understood. From the perspective of modeling the migration behavior of grain boundaries under irradiation, it seems that $\alpha_{v, i}$ represents the behavior of a boundary subjected to unequal fluxes of point defects within the grains adjacent to the boundary. That these unequal fluxes induce unequal segregation profiles at the boundaries is probably not too surprising. But the origin of the unequal fluxes is not known.

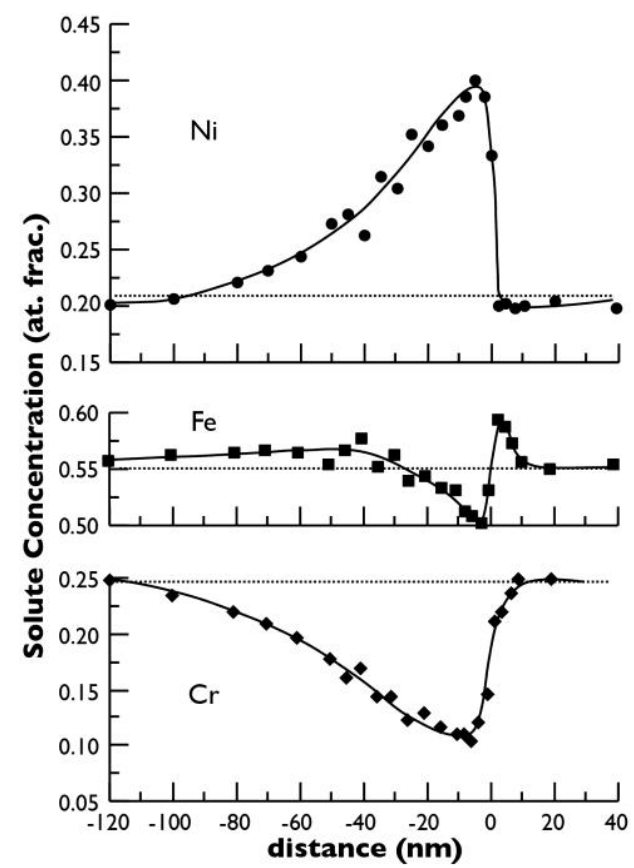

Fig. 5.22 Concentration profiles of $\mathrm{Ni}, \mathrm{Fe}$ and $\mathrm{Cr}$ at moving grain boundaries in a stainless steel irradiated at $420{ }^{\circ} \mathrm{C}$ to a dose of $3.5 \mathrm{dpa}$. The dashed horizontal lines indicate the average concentrations. The atom fractions of $\mathrm{Fe}, \mathrm{Ni}$ and $\mathrm{Cr}$ are normalized to unity. Data of Norris et al. [41]. 


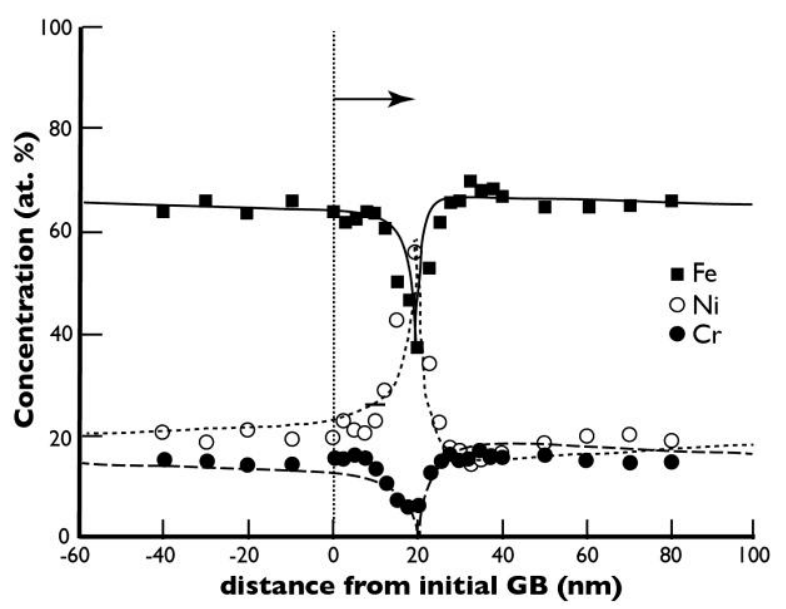

Fig. 5.23. Concentration profiles at a grain boundary that migrated by $\sim 20 \mathrm{~nm}$ during electron irradiation of an austenitic stainless steel at $400{ }^{\circ} \mathrm{C}$ to a dose of $14.4 \mathrm{dpa}$. The curves represent the predictions of the model of Sakaguchi et al. [128]. The arrow indicates the direction of migration of the GB.

\subsection{Approaches for Suppressing RIS}

Various strategies have been proposed to reduce or suppress RIS, either by altering solute diffusion or by altering the characteristics of the sinks. We consider a few of these approaches here.

\subsubsection{Effect of Oversized Solute Atoms on RIS}

A first approach has been to use oversize solute atoms to trap point defects, and thus favor point defect recombination over long-range transport. The apparent effectiveness of oversized solute for suppressing RIS was first demonstrated by Kato et al. [129], who doped type 316 stainless steel with approximately 0.35 at. \% Ti, Zr, Hf, V, $\mathrm{Nb}$ or Ta and irradiated specimens using $1 \mathrm{MeV}$ electrons to doses up to $10.8 \mathrm{dpa}$ at temperatures in the range 350 to 500 ${ }^{\circ} \mathrm{C}$. AEM was then used to investigate RIS, and it was shown that RIS of $\mathrm{Cr}$ and $\mathrm{Ni}$ at grain boundaries was essentially eliminated by the additions of $\mathrm{Zr}$ and $\mathrm{Hf}$, though there was no clear correlation between atomic size and the extent to which RIS was reduced. The beneficial effect of $\mathrm{Hf}$ in reducing or eliminating RIS in austenitic steels has been confirmed by Fournier et al. [130] who investigated RIS in Type 316 stainless steel, doped with 0.3 at. \% Hf or Pt. As seen in Fig. 5.24, Pt and Hf additions significantly reduced the RIS of $\mathrm{Cr}$ at $2.5 \mathrm{dpa}$, but the suppression of RIS of $\mathrm{Cr}$, $\mathrm{Ni}$ and $\mathrm{Fe}$ in the Hf-containing steel was not as large at a dose of $5 \mathrm{dpa}$ as it was at $2.5 \mathrm{dpa}$ (the Pt-containing steel was not irradiated to $5 \mathrm{dpa}$ ). 


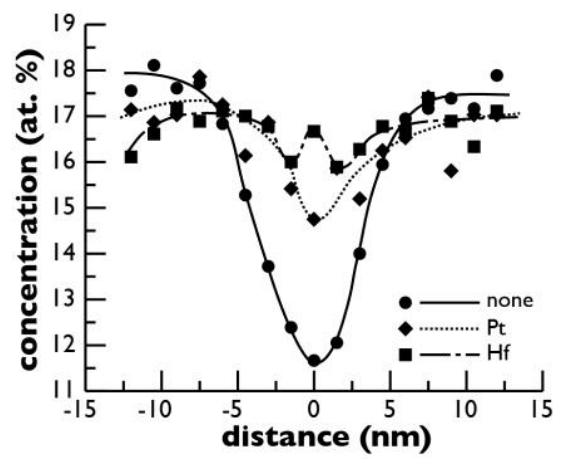

Figure 5.24. The effect of the oversized solute atoms Hf and $\mathrm{Pt}$ on the segregation of $\mathrm{Cr}$ in an austenitic stainless steel irradiated at $400{ }^{\circ} \mathrm{C}$ to 2.5 dpa using energetic protons. Data of Fournier et al. [130].

Subsequent research by Hackett et al. [131] purports to show that oversized $\mathrm{Zr}$ is especially effective in reducing the depletion of $\mathrm{Cr}$ at grain boundaries in proton-irradiated Type 316 stainless steel, but the published data indicate that for the most part the effect is small and dependent on the dose and irradiation temperature. The only significant effect of $\mathrm{Zr}$ is seen in an alloy irradiated at $400{ }^{\circ} \mathrm{C}$ to a dose of $3 \mathrm{dpa}$; otherwise there does not appear to be a major difference between the potency of $\mathrm{Hf}$ and $\mathrm{Zr}$ on the RIS of $\mathrm{Cr}$ at GBs. First-principles ab initio calculations by Hackett et al. [132] indicate that the binding energy between Hf atoms and vacancies is much smaller than that of $\mathrm{Zr}$ and vacancies. The authors claim that this is consistent with the greater potency of $\mathrm{Zr}$ in affecting the RIS of $\mathrm{Cr}$, and indeed it might account for the behavior observed under some irradiation conditions. Yabuuchi etal. [133] investigated the influence of oversized elements on the thermal stability of vacancies in type 316L stainless steels using positron annihilation spectroscopy. They found that vacancies in "pure" 316L stainless steels were mobile at $300{ }^{\circ} \mathrm{C}$ but were immobilized by doping of $\mathrm{Zr}$, Ti, $\mathrm{Nb}$ or Hf. They suggest that the vacancy mobility is inhibited by interactions with oversize solute atoms, but their results do not differentiate the potency of the oversized solute atoms investigated. Overall, we also would expect that, if the oversized solute atoms can migrate sufficiently, they would probably start clustering, in which case their effectiveness to suppress RIS would decrease over time. This conjecture is consistent with the aforementioned dose effect reported by Fournier et al. [130].

There do not appear to be many investigations of the effect of oversized solutes on the suppression of RIS in ferritic and F/M steels. We are aware of one such study by Lu et al. [134], which showed that the addition of 1 at. \% Hf to a F/M (9.2 Cr, 1.0 Mo, 0.7 Nb, 1.0 W, all wt.\%) suppressed RIS of $\mathrm{Cr}$ and $\mathrm{P}$ in specimens irradiated at $300{ }^{\circ} \mathrm{C}$ using $200 \mathrm{keV} \mathrm{Ni}^{+}$ions to $0.3 \mathrm{dpa}$. 
Additions of $\mathrm{Hf}$ thus reduce or eliminate RIS in both austenitic and ferritic steels irradiated to relatively low doses.

\subsubsection{The Role of Interstitial Atoms on RIS}

Another approach to suppress RIS, in particular in steels has been the addition of interstitial alloying elements. In particular, Kano et al. [135] investigated the RIS of Cr, Ni and $\mathrm{Si}$ in two austenitic steels similar to Types 304 and 316 . The 316 alloys contained $0.004,0.019$ and $0.052 \% \mathrm{C}$ and the 304 alloys contained $0.003,0.032$ and $0.095 \% \mathrm{~N}$. The changes in concentration at the grain boundaries compared to their values in the bulk were small, but appear to have been systematic, especially regarding the concentrations of $\mathrm{Ni}$ and the impurity element $\mathrm{Si}$, both of which segregate to grain boundaries during irradiation. The main results are shown in Fig. 5.25 where it is evident that the influence of interstitial $\mathrm{C}$ and $\mathrm{N}$ is most pronounced on the RIS of Ni, with rather smaller effects on the $\mathrm{Cr}$ and Si concentrations. Kano et al. [135] did not attempt to explain their results, but noted that the nucleation of dislocation loops was enhanced by the interstitial additions.
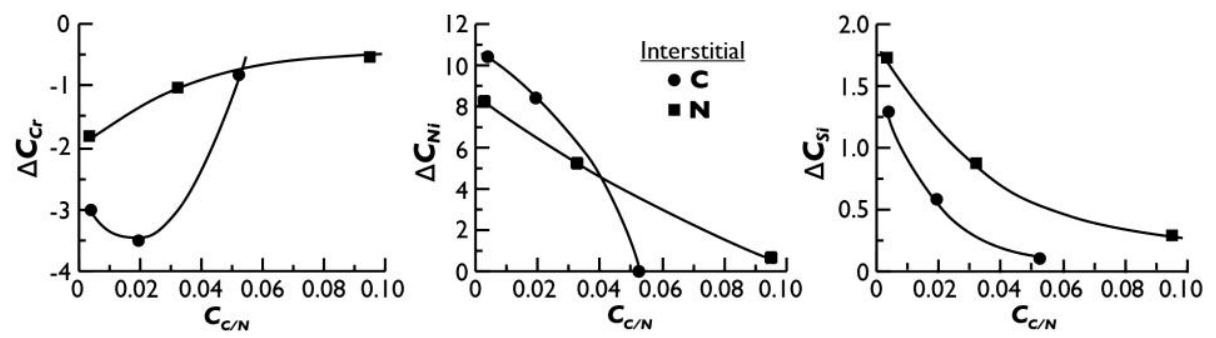

Figure 5.25. The effect of alloying with interstitial $\mathrm{C}$ and $\mathrm{N}$ atoms on RIS in an austenitic steel irradiated by $12 \mathrm{MeV} \mathrm{Ni}^{3+}$ ions at $300{ }^{\circ} \mathrm{C}$. All units of concentration are in wt. \%. The abcissae represent the changes in concentration of the specified solute atom $(\mathrm{Cr}, \mathrm{Ni}, \mathrm{Si})$ measured at the half-widths of the concentration profiles at the GBs, referred to the concentrations far from the boundaries. Data of Kano et al. [135].

\subsubsection{The Effect of Grain Boundary Structure and Character on RIS}

A third aspect of strategies for RIS suppression is to control the efficiency of grain boundaries as sinks for point defects. The character of a grain boundary, specifically whether it is a low-angle boundary consisting of a wall of dislocations, or whether it is a special boundary, such as a coherent twin boundary or another special boundary separating grains with a small 
number of coincident lattice sites (CSL), has been shown experimentally to affect RIS [136,137]. The first observations were made on proton irradiated and high-voltage electron irradiated austenitic stainless steels, respectively, irradiated at temperatures in the range 350 to $450{ }^{\circ} \mathrm{C}$. Both investigations showed that the extent of RIS, characterized by the depletion of $\mathrm{Cr}$ and enrichment of $\mathrm{Ni}$, was significantly reduced at low-angle grain boundaries and special boundaries of the type $\Sigma 3$ (coherent twin boundaries) and 29 . An example, taken from the work of Sakaguchi et al. [138], is shown in Fig. 5.26 for grain boundary orientations symmetric around a $\langle 110\rangle$ axis of rotation. Additional research by Kai et al. [139] has shown that the magnitude of Cr depletion at special boundaries in irradiated Type 304 SS increases as $\Sigma$ increases. Their experiments and model calculations also show that the widths of the segregation fields increase as $\Sigma$ increases.

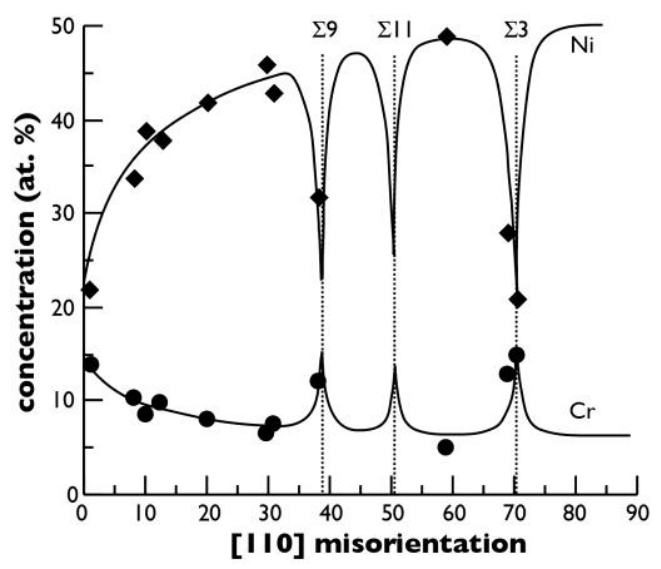

Figure 5.26. The effect of grain boundary misorientation on RIS of $\mathrm{Cr}$ and $\mathrm{Ni}$ in an austenitic stainless steel irradiated to a dose of $3 \mathrm{dpa}$ at $350{ }^{\circ} \mathrm{C}$. Data of Sakaguchi et al. [138].

The qualitative explanation for the data seen in Fig. 5.26 is clear. Low-angle and special boundaries are poor sinks for point defects, so the gradients of vacancies and interstitials in their vicinities become increasingly small as the ideal CSL boundary orientations are approached. Consequently the fluxes of point defects decrease dramatically, hence preferential diffusion of solute by any mechanism is eliminated. In this context it is interesting to point out that Tomozawa et al. [116] did not observe any solute segregation or depletion to $\Sigma 3$ boundaries in their unirradiated specimens, cf. the segregation shown in Fig. 5.26. Sakaguchi et al. [140] have attempted to exploit this behavior by a grain-boundary engineering approach that attempts to incorporate large numbers of $\Sigma 3$ boundaries. 
Modeling of the dependence of RIS on grain boundary character requires the introduction of a parameter that captures the strength of the boundary as a point-defect sink. Models that do this successfully have been developed by Duh et al. [141] and Sakaguchi et al. [128]. Both models are based on the WOL theory and reproduce the observed dependence of RIS on grain boundary misorientation with reasonable accuracy. The curves in Fig. 5.26 show the results of the calculations of Sakaguchi et al. [138]. The agreement between the calculated dependencies of the concentrations of $\mathrm{Cr}$ and $\mathrm{Ni}$ is excellent. The agreement between the predictions of their theory and the data of Duh et al. [136] is also excellent.

Just as it does in austenitic steels, GB misorientation impacts RIS in ferritic steels. Field et al. [142,143] find that RIS of Cr at low-angle or special boundaries is much lower than at general GB, and generally increases with increasing GB misorientation. Field et al. present a rate-theory model that captures the observed experimental behavior.

\subsection{TNES and W-Shaped Concentration Profiles}

The search for a satisfactory explanation for the W-shaped concentration profiles, exemplified by the data in Fig. 5.17 (1 dpa), has proven to be vexing and elusive. As noted, such profiles have been observed in both austenitic and ferritic stainless steels $[117,118]$, see for instance the Ni concentration profile in Fig. 5.21. More recently, Marquis et al. [144] observed $\mathrm{W}$-shaped Cr-concentration profiles at a specific GB in a ferritic steel (15.1 at. \% $\mathrm{Cr})$. The segregation of $\mathrm{Cr}$ and $\mathrm{C}$ prior to irradiation and at a dose of $\sim 0.5 \mathrm{dpa}$ is shown in Fig. 5.27. Considerable TNES of Cr was found (3 to 4 at. \% Cr, as was TNES of C (almost 2 at. \%), even though the concentration of $\mathrm{C}$ in the steel was less than $10 \mathrm{wppm}$. The authors suggest that the TNES of $\mathrm{C}$ at the GB helps to stabilize the RIS of $\mathrm{Cr}$ to the boundary. They also observe that the concentration of $\mathrm{Cr}$ that segregates to the boundary decreases with increasing dose [145]. The concentration profile of $\mathrm{Cr}$ is clearly $\mathrm{W}$-shaped after irradiation to $0.5 \mathrm{dpa}$, and while the authors claim that this profile is evolving to a $\mathrm{V}$ shape, the only results they show to this effect are in a heavy-ion irradiated ODS-strengthened steel [125]. The maximum fluence in this work was a factor of 3 higher than used previously [144], but the irradiation temperature was also higher $\left(500\right.$ cf. $\left.350^{\circ} \mathrm{C}\right)$.

We believe that the authors correctly attribute the persistence of the $\mathrm{W}$-shaped $\mathrm{Cr}$ concentration profile to stabilization by the prior TNES of carbon to the grain boundary. 
Additional support for the possible role of interstitials is provided by Allen et al. [146], who suggested that the interaction of $\mathrm{Cr}$ with interstitial alloying elements $\mathrm{B}, \mathrm{C}$ and $\mathrm{N}$, or the ordering tendency of $\mathrm{Ni}$ and $\mathrm{Cr}$, might be important in producing the $\mathrm{W}$-shaped profile. Additional evidence for this conjecture is seen in the Si concentration profiles reported by Kano et al. [135] in their ultra-low nitrogen and ultra-low carbon austenitic steel. Adding to the mystery, however, is the disappearance of the $\mathrm{W}$-shaped Si concentration profiles in the steels with larger interstitial concentrations.
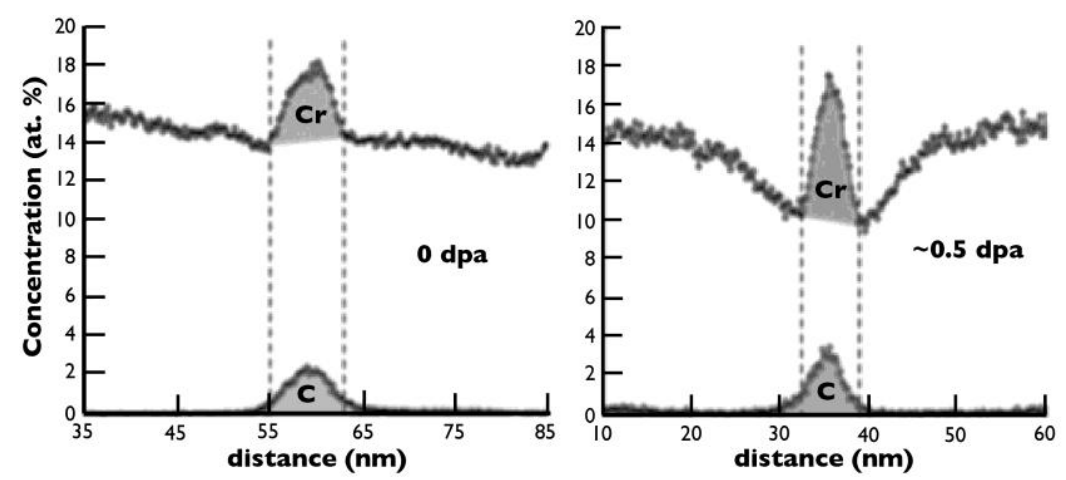

Figure 5.27. $\mathrm{Cr}$ and $\mathrm{C}$ concentration profiles across the grain boundary as function of depth from the implanted surface in a ferritic steel self-ion irradiated at $350{ }^{\circ} \mathrm{C}$. The unirradiated alloy, 0 $\mathrm{dpa}$, is from the specimen characterized beyond the end of the ion range. The concentration profile at 0.5 dpa was taken from the alloy examined at a depth of $300 \mathrm{~nm}$. Data of Marquis et al. [144].

It is not possible to reproduce this behavior using models that invoke only the IKE, as is evident in the modeling work of Marwick et al. [147], Perks and Murphy [81] and Allen and Was [83]. Figure 5.28 (a) illustrates the expected $\mathrm{Cr}$ concentration profile arising from a flux of vacancies to grain boundaries under intermediate cooling conditions. Since $\mathrm{Cr}$ is the fastest diffusing element in austenitic stainless steels [148], a flux of vacancies towards the grain boundary will induce a $\mathrm{Cr}$ flux in the opposite direction, leading to depletion of $\mathrm{Cr}$ rather than enrichment at the GB by TNES. Nevertheless, there are diffusion-based theoretical and modeling efforts that have had at least a modicum of success. In particular, Nastar [149] has reproduced a W-shaped profile using the theory of Grandjean et al. [65], though quantitative agreement with the experimentally observed profile is not very good. She also shows that the pre-existing profile produced by TNES is not crucial in the formation of the W-shaped profile. Additionally, Soisson [150] has performed KMC simulations that produce a W-shaped profile at a GB in an irradiated ideal solid solution of a body centered cubic alloy containing $10 \%$ A, $90 \%$ B. He has also 
shown definitively that non-monotonic concentration profiles can be reproduced using the WOL theory with an appropriate choice of physical parameters and boundary conditions. An example of these results are seen in Fig. 5.28. The array of dots in Fig. 5.28 represents the scatter in the data on simulation and the solid curve represents the calculations using the WOL theory; the agreement is exceptionally good.
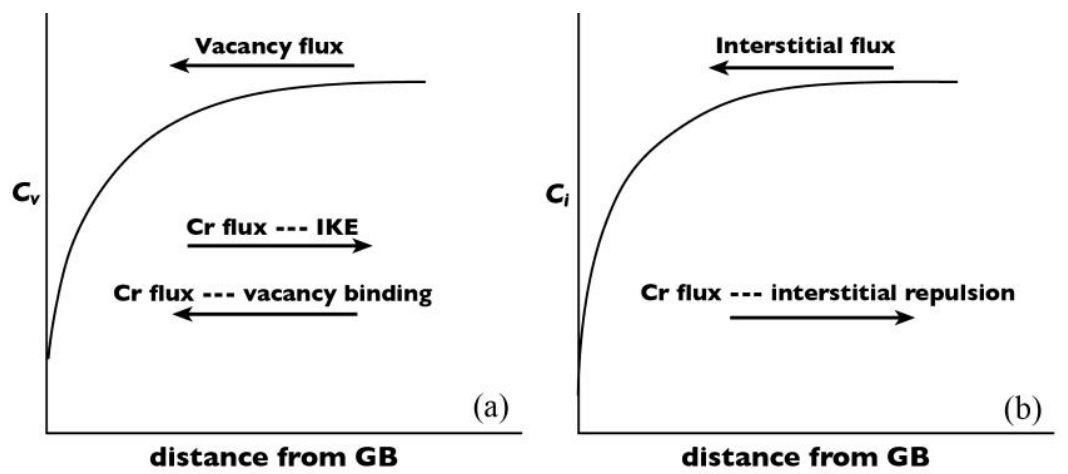

Figure 5.28. Schematic illustration of the segregation tendencies of Cr. In (a) the flux of Cr from the IKE is opposite to that if $\mathrm{Cr}$ atoms are bound to vacancies, as postulated by Faulkner et al. [151]. In (b) The flux of $\mathrm{Cr}$ is opposite to that of interstitials according to the theory of Faulkner et al.

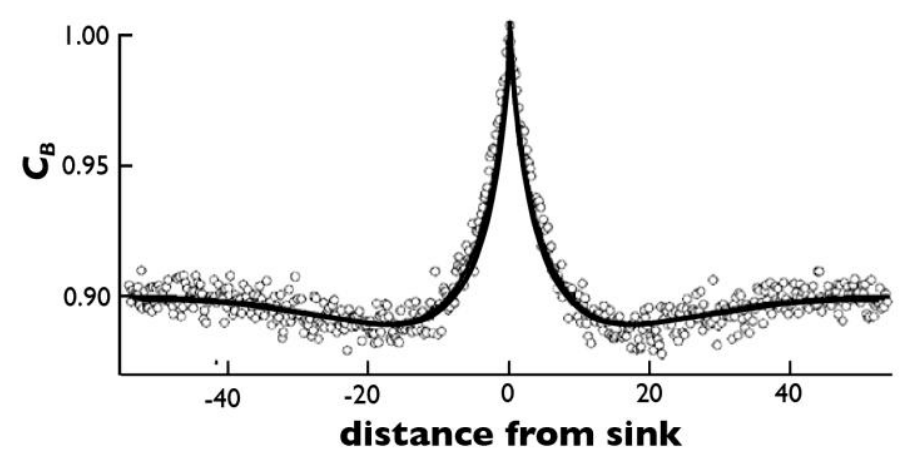

Figure 5.29. Results of the KMC simulations of Soisson [150] for an A-B ideal solid solution irradiated at $500 \mathrm{~K}$ to a dose of $0.5 \mathrm{dpa}$ using a dose rate of $10^{-3} \mathrm{dpa} / \mathrm{s}$. In the simulations $d_{A v}>$ $d_{B v}$ and $d_{A i}=d_{B i}$. The scatter in the simulations is illustrated by overlapping multiple profiles and the solid curve shows the results of the calculations according to the theory of Wiedersich et al. [62].

Rate theory models are capable of reproducing some of the features of W-shaped profiles, but there is another type of model that is also capable of predicting these profiles. It is a model by Goodwin et al. [36], which assumes that solute atoms are strongly bound to vacancies or interstitials and migrate to sinks accordingly. The model of Goodwin et al. combines Faulkner's 
theory of TNES [152] and the theory of Faulkner et al. [151] on RIS. In the TNES theory of Faulkner, oversized $\mathrm{Cr}$ atoms have a positive binding energy with vacancies and a negative binding energy with interstitials. TNES leads to enrichment of $\mathrm{Cr}$ at $\mathrm{GBs}$ since vacancy-Cr complexes diffuse towards them during cooling, as illustrated in Fig. 5.28(a). During irradiation the flux of interstitials towards grain boundaries exceeds the vacancy flux due to their higher mobility. In the theory of RIS of Faulkner et al. [151] interstitial Ni-Ni or Ni-Fe mixed dumbbells are driven towards grain boundaries, thereby producing a flux of $\mathrm{Cr}$ in the opposite direction, as illustrated schematically in Fig. 5.28(b). This theory therefore also predicts the RIS of undersized Ni atoms at grain boundaries. At an intermediate stage of irradiation the initially enhanced concentration of $\mathrm{Cr}$, due to TNES, becomes depleted by the flux of $\mathrm{Cr}$ away from the GB producing a $\mathrm{W}$-shaped concentration profile. Subsequent irradiation depletes all the $\mathrm{Cr}$ from the GB and the profile becomes monotonic with distance from the GB. Faulkner, Goodwin and co-workers do not address the segregation of $\mathrm{Ni}$ and $\mathrm{Fe}$, so it is not known what kind of segregation behavior their model would predict. Unlike the theory of Nastar [149], TNES is an essential ingredient of the model of Goodwin et al. [36].

There have been no attempts at modeling the influence of dissolved interstitial atoms on the segregation behavior of substitutional solutes in any alloy system, so far as we are aware. We also note that $\mathrm{W}$-shaped profiles are usually transient ones, and that one single mechanism (TNES driven by vacancies, RIS driven by vacancies, or RIS driven by interstitials) typically cannot correctly reproduce these profiles, and so most explanations will require two competing mechanisms. The formation, shapes and lifetimes of $\mathrm{W}$-shaped profiles remain open challenges for theory and modeling.

\section{Closing Comments}

Radiation induced segregation remains an important practical issue because of its impact on the mechanical properties of the materials where RIS takes place. In many ways, RIS is a mature and well-understood problem. As illustrated in this review, a large body of work employing experiments, simulations, and modeling, has made it possible, in the vast majority of cases, to identify the individual mechanisms responsible for RIS, typically resulting from the coupling of chemical and point defect fluxes, and their interactions with characteristics of sinks and the material microstructure. Despite this remarkable progress, there are still unanswered 
questions which provide the impetus for further improving our ability to predict and mitigate RIS in specific materials, especially for materials of direct interest to the nuclear industry. One aspect of this challenge relates to the complexity of these materials, which are typically multicomponent, non-dilute alloys, often with multiple phases, so as to optimize properties such as ductility and strength at both low and elevated temperatures, creep resistance, and corrosion resistance. In these materials, RIS often results from a delicate balance between competing or synergistic processes, for instance vacancy-driven and interstitial-driven solute transport. In order to mitigate or predict RIS, it is therefore necessary to take into account, and in fact embrace, this material complexity.

A second aspect of the challenges posed by RIS is that it is driven by non-equilibrium processes, set in by irradiation. As a result, the microstructural response of the material does not need to follow equilbrium thermodynamics, and in fact it rarely does, often leading to wellidentified non-equilibrium phase transitions such as radiation-induced precipitation in undersaturated alloys and microstructural self-organization reactions. In order to meet these challenges, our community will be able to draw from unparalleled advances in experimental characterization techniques with nanometer scale structural and chemical resolutions, such as aberration corrected transmission electron microscopy and atom probe tomography, as well as new developments in modeling, from the atomistic scale through first principles calculations to the continuum scale through approaches like the SCMF kinetic model.

The experimental results reported in this review illustrate clearly the challenges faced by future modeling techniques and numerical simulations. A first point is that, owing to the broad spectrum of length scales and time scales involved in RIS, brute force atomistic simulations by molecular dynamics or by kinetic Monte Carlo techniques will not suffice. MD simulations, even using accelerated algorithms, cannot capture long diffusional time scales, while KMC simulations require an a priori identification of the possible elementary events contributing to microstructural evolution, and KMC cannot handle stress and relaxation effects in large systems. Continuum models, in the spirit of the WOL model and of the SCMF, require clear physical derivations of their evolution equation, and their parametrization requires a fair amount of atomistic information which is specific to the alloy systems of interest. It is thus clear that predictive modeling in the future will require a multiscale approach that integrates atomistic methods and continuum models. This integration of distinct methods to address mesoscale 
evolutions of materials is in fact a well recognized challenge by itself in many other areas of Materials Science.

Beyond the issue of integrating different modeling and simulations techniques additional, specific challenges should be met for RIS modeling to achieve its full predictive power. A first important challenge concerns the case of concentrated alloys, which are commonly used for applications, e.g., austenitic and ferritic/martensitic stainless steels. For these alloys, the number of distinct jump frequencies is too large for direct calculations by first principles methods, and some means needs to be devised to estimate as accurately as possible the effect of local atomic environments on jump frequencies. One approach developed within the SCMF method is to rely on mean field approximations, see for instance [153]. Another approach is to use genetic algorithms and neural networks [154-156]. More work is however needed to further develop these approaches before they can be routinely used for RIS modeling. A second modeling challenge is the inclusion of small defect clusters. These small clusters, which form either directly in displacement cascades or by reaction between isolated point defects, can contribute significantly to the evolution of the point defect population, reducing the concentration of socalled "freely migrating" defects, but they can also directly transport solute atoms very effectively, as for instance in the case of small interstitial clusters migrating unidimensionally [157-159]. These effects are not included in existing RIS models. The development of practical and predictive RIS models for the alloys of interest to the nuclear industry will require the integration of many if not all of the above factors, including multicomponent alloys, non-dilute concentrations, stress and strain, defect clustering, into models that, despite their inherent complexity will be robust and numerically efficient. As discussed in this review, recent developments in theory and modeling have helped researchers to make remarkable progress. Despite the daunting complexities of RIS and its numerous and varied consequences, it is expected that this positive trend will continue unabated.

In addition to the aforementioned active research on radiation-induced segregation, it is also worth noting that efforts have been put into the development of materials that would be immune from RIS. One can envision designing such materials by relying on microstructures with a high density of point-defect traps and sinks to facilitate point-defect recombination, thus minimizing the amount of chemical segregation to any given sink. This strategy has been recognized decades ago [160], but it has received increased interest thanks to advances in 
materials fabrication techniques that are making it possible to synthesize materials for nuclear applications with nanometric grain size [161], with high density of structural and chemical interfaces [162], or with high number density of small refractory precipitates $[163,164]$. The main challenge in these approaches is to ensure that the high, initial, sink density is not lost during irradiation through grain boundary migration, dislocation glide and climb, and precipitate coarsening. One possible approach to overcome this issue is to rely on the self-organization of the microstructure under irradiation $[165,166]$, as illustrated here in section 5.1. Selforganization could then yield microstructures that, by design, contain a high number density of stable sinks under irradiation [167]. More work is however needed to validate this approach, and to evaluate its compatibility with other design requirements for nuclear materials.

\section{Acknowledgments}

Work by PB was supported by the U.S. Department of Energy (DOE), Office of Science, Basic Energy Sciences (BES) under Award \# DEFG02-05ER46217. 


\section{REFERENCES}

1. Ardell AJ: Radiation-induced solute segregation in alloys. In: Ghetta V, Gorse D, Maziere D, Pontikis V editors, Proceedings of the NATO Advanced Study Institute on Materials Issues For Generation IV Systems: Status, Open Questions and Challenges, Dordrecht: Springer Netherlands, 2008:285-310.

2. Nastar M, Soisson F: Radiation-induced segregation. In: Konings RJM editor, Comprehensive Nuclear Materials, Vol 1: Basic Aspects of Radiation Effects In Solids/Basic Aspects of MultiScale Modeling, Amsterdam: Elsevier, 2012:471-496.

3. Okamoto PR, Rehn LE: Radiation-induced segregation in binary and ternary alloys. $\mathrm{J} \mathrm{Nucl}$ Mater 1979, 83:2-23.

4. Martin G, Bellon P: Driven alloys. Sol State Phys - Advances in Research and Applications 1997, 50:189-331.

5. Fukuya K: Current understanding of radiation-induced degradation in light water reactor structural materials. J Nucl Sci Tech 2013, 50:213-254.

6. Zinkle SJ, Was GS: Materials challenges in nuclear energy. Acta Mater 2013, 61:735-758. 7. Allen TR, Kaoumi D, Wharry JP, Jiao Z, Topbasi C, Kohnert A, Barnard L, Certain A, Field KG, Was GS, et al.: Characterization of microstructure and property evolution in advanced cladding and duct: Materials exposed to high dose and elevated temperature. J Mater Res 2015, 30:1246-1274.

8. Marquis EA: Atom probe tomography applied to the analysis of irradiated microstructures. $J$ Mater Res 2015, 30:1222-1230.

9. Okamoto PR, Wiedersich H: Segregation of alloying elements to free surfaces during irradiation. J Nucl Mater 1974, 53:336-345.

10. Barbu A, Ardell AJ: Irradiation-induced precipitation in Ni-Si alloys. Scripta Metall 1975, 9:1233-1237.

11. Rastogi PK, Ardell AJ: Coherent solubilities of $\gamma 2$ in Ni-Al Ni-Si and Ni-Ti alloys. Acta Metall 1969, 17:595-602

12. Kamara A, Ardell AJ, Wagner CNJ: Lattice misfits in four binary Ni-base $\gamma / \gamma 2$ alloys at ambient and elevated temperatures. Metall Mater Trans A 1996, 27:2888-2896.

13. Janghorban K, Ardell AJ: Early stages of irradiation induced $\gamma 2$ precipitation in proton irradiated nickel-silicon alloys. J Nucl Mater 1979, 85-6:719-723.

14. Rehn LE, Okamoto PR, Potter DI, Wiedersich H: Effect of solute misfit and temperature on irradiation-induced segregation in binary Ni-alloys. J Nucl Mater 1978, 74:242-251.

15. Ardell AJ, Janghorban K: Irradiation-induced phase transformations in binary Ni and Pd base alloys. In: Nolfi FV editor, Phase Transformations During Irradiation, New York: Applied Science Publishers, 1983:291-329.

16. Weaver $\mathrm{L}$, Ardell AJ: The formation of $\mathrm{Pd}_{8} \mathrm{~W}$ in proton irradiated palladium-rich $\mathrm{Pd}-\mathrm{W}$ alloys. Scripta Metall 1980, 14:765-768.

17. Mostafa M, Ardell AJ: The formation of $\mathrm{Pd}_{8} \mathrm{Mo}$ in proton-irradiated Pd-Mo solid-solutions. Mater Lett 1987:67-70.

18. Cheng J, Ardell AJ: On the stability of the ordered $\mathrm{Pd}_{8} \mathrm{~V}$ phase in a proton-irradiated $\mathrm{Pd}$ 15at.\%V alloy. J Less-Comm Metals 1988:45-53.

19. Lesueur D: Amorphisation sous irradiation d'un alliage palladium-silicium, C R Acad Sci Paris 1968, 266:1038-1041. 
20. Thomas G, Mori H, Fujita H, Sinclair R: Electron-irradiation induced crystalline amorphous transitions in Ni-Ti alloys. Scripta Metall 1982, 16:589-592.

21. Parsons JR, Balluffi RW: Displacement spike crystallization of amorphous germanium during irradiation. J Phys Chem Solids 1964, 25:263-272.

22. Azam N, Lenaour L, Rivera C, Grosjean P, Sacovy P, Delaplace J: Crystallization and irradiation effects of amorphous $\mathrm{Fe}_{40} \mathrm{Ni}_{38} \mathrm{Mo}_{4} \mathrm{~B}_{18}$ alloy. J Nucl Mater 1979, 83:298-304.

23. Rehn LE, Okamoto, PR: Non-equilibrium segregation in irradiated alloys. In: Nolfi FV editor, Phase Transformations During Irradiation, New York: Applied Science Publishers, 1983:247-290.

24. Barbu, A: Précipitation induite et gonflement dans la solution solide sous-saturée Ni $6 \%$ at Ge irradiée aux electrons de $1 \mathrm{MeV}$. In; Poirier P, Dupouy JM editors, Comportement sous Irradiation des Materiaux Métalliques et des Coeurs des Réacteurs Rapide, Gif-sur-Yvette: Commissariat à l'Energie Atomique, 1979:65-73.

25. Gupta RP, Lam NQ: Defect-solute interactions and radiation-induced segregation in Ni(Si) and $\mathrm{Ni}(\mathrm{Ge})$ alloys. Scripta Metall 1979, 13:1005-1010.

26. Chakin V, Kazakov V, Goncharenko Y, Ostrovsky Z: Formation of the $\sigma$-phase in Cr-Fe alloys under irradiation. Journal of Nuclear Materials 1996, 233:573-576.

27. Ghosh G, Olson GB: The isotropic shear modulus of multicomponent Fe-base solid solutions. Acta Mater 2002, 50:2655-2675.

28. Erck RA, Rehn LE: Kinetics of radiation-induced segregation in molybdenum-rhenium alloys. J Nucl Mater 1989, 168:208-219.

29. King HW: Quantitative size-factors for metallic solid solutions. J Mater Sci 1966, 1:79-90. 30. Meslin E, Radiguet B, Loyer-Prost M: Radiation-induced precipitation in a ferritic model alloy: An experimental and theoretical study. Acta Mater 2013, 61:6246-6254.

31. Wakai E, Hishinuma A, Kato Y, Yano H, Takaki S, Abiko K: Radiation-induced $\alpha 2$ phase formation on dislocation loops in $\mathrm{Fe}-\mathrm{Cr}$ alloys during electron irradiation. J Phys IV, Colloque C7, 1995, 5:277-286.

32. Bhattacharya A, Meslin E, Henry J, Pareige C, Decamps B, Genevois C, Brimbal D, Barbu A: Chromium enrichment on the habit plane of dislocation loops in ion-irradiated high-purity FeCr alloys. Acta Mater 2014, 78:394-403.

33. Marinelli P, Baruj A, Guillermet AF, Sade M: Lattice parameters of metastable structures in quenched Fe-Mn alloys. Part I: Experimental techniques, bec and fcc phases. Z Metallkde 2000, 91:957-962.

34. Aust KT, Hanneman RE, Niessen P, Westbrook JH: Solute induced hardening near grain boundaries in zone refined metals. Acta Metall 1968, 16:291-302.

35. Westbrook JH, Aust KT: Solute hardening at interfaces in high-purity lead-I Grain and twin boundaries. Acta Metall 1963, 11:1151-1163.

36. Goodwin CC, Faulkner RG, Fisher SB: In: Nanstad RK, Hamilton ML, Garner FA, Kumar SA editors, The modelling of combined radiation-induced and thermal non-equilibrium segregation of chromium in neutron-irradiated austenitic stainless steels. Effects of Radiation on Materials: $18^{\text {th }}$ International Symposium. ASTM STP 1325, 1999:634-646.

37. Howard RE, Lidiard AB: Annealing of vacancies in dilute alloys. Philos Mag 1965, 11:11791187.

38. Anthony TR, Hanneman RE: Non-equilibrium segregation of impurities in quenched dilute alloys. Scripta Metall 1968, 2:611-614. 
39. Anthony TR: Solute segregation in vacancy gradients generated by sintering and temperature changes. Acta Metall 1969, 17:603-609.

40. Marwick AD: Segregation in irradiated alloys: inverse Kirkendall effect and effect of constitution on void swelling. J Phys F-Metal Phys 1978, 8:1849-1861.

41. Norris DIR, Baker C, Taylor C, Titchmarsh JM: In: Stoller, RE, Kumar, AS, Gelles, DS editors, Radiation-induced segregation in $20 \mathrm{Cr} / 25 \mathrm{Ni} / \mathrm{Nb}$ stainless steel. Effects of Radiation on Materials: $15^{\text {th }}$ International Symposium. ASTM STP 1125, 1992:603-620.

42. Wiedersich H, Lam NQ: Theory of radiation-induced segregation. In: Nolfi FV editor, Phase Transformations During Irradiation, New York: Applied Science Publishers, 1983:1-46.

43. Howard RE, Manning JR: Kinetics of solute-enhanced diffusion in dilute face-centered-cubic alloys. Phys Rev 1967, 154:561-568.

44. Robrock, K-H: The interaction of self-interstitial atoms with solute atoms in metals. In: Nolfi FV editor, Phase Transformations During Irradiation, New York: Applied Science Publishers, 1983:115-146.

45. Johnson RA, Lam NQ: Solute segregation in metals under irradiation. Phys Rev B 1976, 13:4364-4375.

46. Barbu A: Expressions microscopiques des flux de solute et d'interstitiels dans des gradients de concentration de solute et d'interstitiels pour un cristal de structure c.f.c. Acta Metall 1980, 28:499-506.

47. Bardeen J, Herring C: In: Shockley W editor, Imperfections in Nearly Perfect Crystals, American Society for Metals, Cleveland, OH; 1951:87-111.

48. Philibert J: Atom Movements: Diffusion and Mass Transports in Solids. Monographies de Physique: Éditions de Physique, Les Ulis, France 1991.

49. Allnatt AR, Lidiard AB: Atomic Transport in Solids. Cambridge University Press; 1993.

50. Manning JR: Vacancy-wind effect in diffusion and deviations from thermodynamic equilibrium conditions. Can J Phys 1968, 46:2633-2643.

51. Nastar M, Dobretsov VY, Martin G: Self-consistent formulation of configurational kinetics close to equilibrium: the phenomenological coefficients for diffusion in crystalline solids. Philos Mag A 2000, 80:155-184.

52. Nastar M: A mean field theory for diffusion in a dilute multi-component alloy: a new model for the effect of solutes on self-diffusion. Philos Mag 2005, 85:3767-3794.

53. Nastar M: Diffusion and coupled fluxes in concentrated alloys under irradiation: a selfconsistent mean-field approach. Compt Rend Phys 2008, 9:362-369.

54. Ducastelle F: Order and phase stability in alloys. In Interatomic Potential and Structural Stability. Springer Series in Solid-State Sciences Vol.114, 1993:133-142.

55. Garnier T, Nastar M, Bellon P, Trinkle DR: Solute drag by vacancies in body-centered cubic alloys. Phys Rev B 2013, 88:134201.

56. Barbe V, Nastar M: Split interstitials in an interacting bec alloy. I. Extension of the cluster variation method. Phys Rev B 2007, 76:054205.

57. Barbe V, Nastar M: Split interstitials in an interacting bec alloy. II. Transport coefficients. Phys Rev B 2007, 76:054206.

58. Garnier T, Manga VR, Trinkle DR, Nastar M, Bellon P: Stress-induced anisotropic diffusion in alloys: Complex Si solute flow near a dislocation core in Ni. Phys Rev B 2013, 88:134108. 59. Garnier T, Li ZB, Nastar M, Bellon P, Trinkle DR: Calculation of strain effects on vacancymediated diffusion of impurities in fcc structures: General approach and application to $\mathrm{Ni}_{1-\mathrm{x}} \mathrm{Si}_{\mathrm{x}}$. Phys Rev B 2014, 90:184301 
60. Garnier T, Manga VR, Bellon P, Trinkle DR: Diffusion of Si impurities in Ni under stress: A first-principles study. Phys Rev B 2014, 90:024306.

61. Manning JR: Correlation factors for diffusion in nondilute alloys. Phys Rev B 1971, 4:11111121.

62. Wiedersich H, Okamoto PR, Lam NQ: Theory of radiation-induced segregation in concentrated alloys. J Nucl Mater 1979, 83:98-108.

63. English CA, Murphy SM, Perks JM: Radiation-induced segregation in metals. J Chem SocFaraday Trans 1990, 86:1263-1271.

64. Wolfer WG: Drift forces on vacancies and interstitials in alloys with radiation-induced segregation. J Nucl Mater 1983, 114:292-304.

65. Grandjean Y, Bellon P, Martin G: Kinetic-model for equilibrium and nonequilibrium segregation in concentrated alloys under irradiation. Phys Rev B 1994, 50:4228-4231.

66. Martin G: Atomic mobility in Cahn's diffusion-model. Phys Rev B 1990, 41:2279-2283.

67. Lam NQ, Kumar A, Wiedersich H: Kinetics of radiation-induced segregation in ternary alloys. In Brager HR, Perrin JS editors, Effects of Radiation on Materials: $11^{\text {th }}$ International Symposium ASTM STP 782; 1982:985-1007.

68. Averback RS, Rehn LE, Wagner W, Wiedersich H, Okamoto PR: Kinetics of radiationinduced segregation in Ni-12.7 at.\% Si. Phys Rev B 1983, 28:3100-3109.

69. Okamoto PR, Rehn LE, Averback RS: Solute redistribution processes in ion bombarded alloys. J Nucl Mater 1982, 108:319-330.

70. Van der Ven A, Yu HC, Ceder G, Thornton K: Vacancy mediated substitutional diffusion in binary crystalline solids. Prog Mater Sci 2010, 55:61-105.

71. Messina L, Nastar M, Garnier T, Domain C, Olsson P: Exact ab initio transport coefficients in bec Fe-X (X=Cr, Cu, Mn, Ni, P, Si) dilute alloys. Phys Rev B 2014, 90.

72. Anthony TR: Atom currents generated by vacancy winds. In: Nowick AS, editor, Diffusion in Solids. Academic Press, 1975:353-379.

73. Ardell AJ, Prikhodko S: Coarsening of $\gamma 2$ in Ni-Al alloys aged under uniaxial compression:

II. Diffusion under stress and retardation of coarsening kinetics. Acta Mater 2003, 51:5013-5019.

74. Van der Ven A, Ceder G: First principles calculation of the interdiffusion coefficient in binary alloys. Phys Rev Lett 2005, 94:045901.

75. Van der Ven A, Ceder G, Asta M, Tepesch PD: First-principles theory of ionic diffusion with nondilute carriers. Phys Rev B 2001, 64:184307.

76. Choudhury S, Barnard L, Morgan D, Field K, Allen T, Wharry JP, Jiao Z, Was G, Wirth B: Radiation induced segregation in ferritic-martensitic steels. Trans Amer Nucl Soc 2010, 102:715716.

77. Choudhury S, Barnard L, Tucker JD, Allen TR, Wirth BD, Asta M, Morgan D: Ab-initio based modeling of diffusion in dilute bcc Fe-Ni and $\mathrm{Fe}-\mathrm{Cr}$ alloys and implications for radiation induced segregation. J Nucl Mater 2011, 411:1-14.

78. Barnard L, Tucker JD, Choudhury S, Allen TR, Morgan D: Modeling radiation induced segregation in Ni-Cr model alloys from first principles. J Nucl Mater 2012, 425:8-15.

79. Barnard L, Morgan D: Ab initio molecular dynamics simulation of interstitial diffusion in NiCr alloys and implications for radiation induced segregation. J Nucl Mater 2013.

80. Tucker JD, Najafabadi R, Allen TR, Morgan D: Ab initio-based diffusion theory and tracer diffusion in Ni-Cr and Ni-Fe alloys. J Nucl Mater 2010, 405:216-234. 
81. Perks J, Murphy S: Modelling the major element radiation-induced segregation in concentrated Fe-Cr-Ni alloys. In: Materials for Nuclear Reactor Core Applications, Vol 1. BNES; 1987:165-169.

82. Allen TR, Was GS, Kenik EA: The effect of alloy composition on radiation-induced segregation in Fe-Cr-Ni alloys. J Nucl Mater 1997, 244:278-294.

83. Allen TR, Was GS: Modeling radiation-induced segregation in austenitic Fe-Cr-Ni alloys. Acta Mater 1998, 46:3679-3691.

84. Lam NQ, Janghorban K, Ardell AJ: On the modeling of irradiation-induced homogeneous precipitation in proton-bombarded Ni-Si solid-solutions. J Nucl Mater 1981, 101:314-325.

85. Miller MK: Atom probe tomography characterization of solute segregation to dislocations and interfaces. J Mater Sci 2006, 41:7808-7813.

86. Miller MK: Atom probe tomography characterization of solute segregation to dislocations. Micros Res Tech 2006, 69:359-365.

87. Etienne A, Radiguet B, Pareige P, Massoud JP, Pokor C: Tomographic atom probe characterization of the microstructure of a cold worked 316 austenitic stainless steel after neutron irradiation. J Nucl Mater 2008, 382:64-69.

88. Radiguet B, Etienne A, Pareige P, Sauvage X, Valiev R: Irradiation behavior of nanostructured 316 austenitic stainless steel. J Mater Sci 2008, 43:7338-7343.

89. Toyama T, Nozawa Y, Van Renterghem W, Matsukawa Y, Hatakeyama M, Nagai Y, Al Mazouzi A, Van Dyck S: Irradiation-induced precipitates in a neutron irradiated 304 stainless steel studied by three-dimensional atom probe. J Nucl Mater 2011, 418:62-68.

90. Kuksenko V, Pareige C, Genevois C, Cuvilly F, Roussel M, Pareige P: Effect of neutronirradiation on the microstructure of a Fe-12at.\%Cr alloy. J Nucl Mater 2011, 415:61-66.

91. Meslin E, Radiguet B, Pareige P, Toffolon C, Barbu A: Irradiation-induced solute clustering in a low nickel FeMnNi ferritic alloy. Exper Mech 2011, 51:1453-1458.

92. Jiao Z, Was GS: Precipitate evolution in ion-irradiated HCM12A. J Nucl Mater 2012, 425:105-111.

93. Cauvin R, Martin G: Radiation-induced homogeneous precipitation in undersaturated solidsolutions. J Nucl Mater 1979, 83:67-78.

94. Cauvin R, Martin G: Solid-solutions under irradiation. I. A model for radiation-induced metastability. Phys Rev B 1981, 23:3322-3332.

95. Cauvin R, Martin G: Solid-solutions under irradiation. II. Radiation-induced precipitation in AlZn undersaturated solid-solutions. Phys Rev B 1981, 23:3333-3348.

96. Cauvin R, Martin G: Solid-solutions under irradiation. III. Further comments on the computed solubility limit. Phys Rev B 1982, 25:3385-3388.

97. Martin G: Long-range periodic decomposition of irradiated solid-solutions. Phys Rev Lett 1983, 50:250-252.

98. Badillo A, Bellon P, Averback RS: A phase field model for segregation and precipitation induced by irradiation in alloys. Model Simul Mater Sci Eng 2015, 23:035008.

99. Kenik EA: Radiation-induced segregation in irradiated type-304 stainless-steels. $J$ Nucl Mater 1992, 187:239-246.

100. Etienne A, Radiguet B, Cunningham NJ, Odette GR, Pareige P: Atomic scale investigation of radiation-induced segregation in austenitic stainless steels. J Nucl Mater 2010, 406:244-250. 101. Etienne A, Radiguet B, Pareige P: Understanding silicon-rich phase precipitation under irradiation in austenitic stainless steels. J Nucl Mater 2010, 406:251-256. 
102. Jiao Z, Was GS: Segregation behavior in proton- and heavy-ion-irradiated ferriticmartensitic alloys. Acta Mater 2011, 59:4467-4481.

103. Williams CA, Hyde JM, Smith GDW, Marquis EA: Effects of heavy-ion irradiation on solute segregation to dislocations in oxide-dispersion-strengthened Eurofer 97 steel. $J$ Nucl Mater 2011, 412:100-105.

104. Kuksenko V, Pareige C, Genevois C, Pareige P: Characterisation of Cr, Si and P distribution at dislocations and grain-boundaries in neutron irradiated $\mathrm{Fe}-\mathrm{Cr}$ model alloys of low purity. J Nucl Mater 2013, 434:49-55.

105. Kuksenko V, Pareige C, Pareige P: Intra granular precipitation and grain boundary segregation under neutron irradiation in a low purity Fe-Cr based alloy. J Nucl Mater 2012, 425:125-129.

106. Jiao Z, Was GS: Novel features of radiation-induced segregation and radiation-induced precipitation in austenitic stainless steels. Acta Mater 2011, 59:1220-1238.

107. Hatakeyama M, Tamura S, Yamagata I: Direct observation of solute-dislocation interaction on screw dislocation in a neutron irradiated modified 316 stainless steel. Mater Lett 2014, 122:301-305.

108. Hatakeyama M, Yamagata I, Matsukawa Y, Tamura S: Direct observation of solutedislocation interaction on extended edge dislocation in irradiated austenitic stainless steel. Philos Mag Lett 2014, 94:18-24.

109. Faulkner RG: Segregation to boundaries and interfaces in solids. Int Mater Rev 1996, 41:198-208.

110. Wharry JP, Jiao Z, Shankar V, Busby JT, Was GS: Radiation-induced segregation and phase stability in ferritic-martensitic alloy T 91. J Nucl Mater 2011, 417:140-144.

111. Keast VJ, Williams DB: Grain boundary chemistry. COSSMS 2001, 5:23-30.

110. Wynblatt $\mathrm{P}$, Chatain D: Anisotropy of segregation at grain boundaries and surfaces. Metall Mater Trans A 2006, 37A:2595-2620.

113. Lejcek P, Hofmann S: Thermodynamics of grain boundary segregation and applications to anisotropy, compensation effect and prediction. Crit Rev Sol State Mater Sci 2008, 33:133-163. 114. Miller MK, Yao L: Limits of detectability for clusters and solute segregation to grain boundaries. COSSMS 2013, 17:203-210.

115. Kashinath A, Misra A, Demkowicz MJ: Stable storage of helium in nanoscale platelets at semicoherent interfaces. Phys Rev Lett 2013, 110:086101.

116. Tomozawa M, Miyahara Y, Kako K: Solute segregation on $\Sigma 3$ and random grain boundaries in type 316L stainless steel. Mater Sci Engr A 2013, 578:167-173.

117. Busby JT, Was GS, Bruemmer SM, Edwards DJ, Kenik EA: Influence of initial grain boundary composition on the evolution of radiation-induced segregation profiles. In: Zinkle SJ, Lucas GE, Ewing RC, Williams JS editors, Symposium on Microstructural Processes in Irradiated Materials, 1998 Fall MRS Meeting; 1998, 540:451-456.

118. Was GS, Busby JT, Allen T, Kenik EA, Jenssen A, Bruemmer SM, Gan J, Edwards AD, Scott PM: Emulation of neutron irradiation effects with protons: validation of principle. $J$ Nucl Mater 2002, 300:198-216.

119. Toyama T, Nozawa Y, Van Renterghem W, Matsukawa Y, Hatakeyama M, Nagai Y, Al Mazouzi A, Van Dyck S: Grain boundary segregation in neutron-irradiated 304 stainless steel studied by atom probe tomography. J Nucl Mater 2012, 425:71-75. 
120. Allen TR, Cole JI, Kenik EA, Was GS: Analyzing the effect of displacement rate on radiation-induced segregation in 304 and 316 stainless steels by examining irradiated EBR-II components and samples irradiated with protons. J Nucl Mater 2008, 376:169-173.

121. Muroga T, Yamaguchi A, Yoshida N: Characteristics of radiation-induced solute segregation in candidate and model ferritic alloys. In: Packan NH, Stoller RE, Kumar A editors, Effects of Radiation on Materials: $14^{\text {th }}$ International Symposium, Vol 1, ASTM STP 1046; 1990:396-410.

122. Lu Z, Faulkner RG, Was G, Wirth BD: Irradiation-induced grain boundary chromium microchemistry in high alloy ferritic steels. Scripta Mater 2008, 58:878-881.

123. Wharry JP, Was GS: A systematic study of radiation-induced segregation in ferriticmartensitic alloys. J Nucl Mater 2013, 442:7-16.

124. Wharry JP, Was GS: The mechanism of radiation-induced segregation in ferritic-martensitic alloys. Acta Mater 2014, 65:42-55.

125. Marquis EA, Lozano-Perez S, de Castro V: Effects of heavy-ion irradiation on the grain boundary chemistry of an oxide-dispersion strengthened Fe-12 wt.\% Cr alloy. J Nucl Mater 2011, 417:257-261.

126. Hu R, Smith GDW, Marquis EA: Atom probe study of radiation induced grain boundary segregation/depletion in a Fe-12\%Cr alloy. Prog Nucl Energy 2012, 57:14-19.

127. Wei CC, Aitkaliyeva A, Martin MS, Chen D, Shao L: Microstructural changes of T-91 alloy irradiated by Fe self ions to ultrahigh displacement ratios. Nucl Instr Meth Phys Res Sec B 2013, 307:181-184.

128. Sakaguchi N, Watanabe S, Takahashi H: A new model for radiation-induced grain boundary segregation with grain boundary movement in concentrated alloy system. J Mater Sci 2005, 40:889-893.

129. Kato T, Takahashi H, Izumiya M: Grain-boundary segregation under electron-irradiation in austenitic stainless-steels modified with oversized elements. J Nucl Mater 1992, 189:167-174. 130. Fournier L, Sencer BH, Was GS, Simonen EP, Bruemmer SM: The influence of oversized solute additions on radiation-induced changes and post-irradiation intergranular stress corrosion cracking behavior in high-purity 316 stainless steels. J Nucl Mater 2003, 321:192-209.

131. Hackett MJ, Busby JT, Miller MK, Was GS: Effects of oversized solutes on radiationinduced segregation in austenitic stainless steels. J Nucl Mater 2009, 389:265-278.

132. Hackett MJ, Busby JT, Was GS: The mechanism of $\mathrm{Zr}$ and Hf in reducing radiation-induced segregation in 316 stainless steel. Metall Mater Trans A 2008, 39A:218-224.

133. Yabuuchi A, Maekawa M, Kawasuso A: Influence of oversized elements (Hf, Zr, Ti and

$\mathrm{Nb}$ ) on the thermal stability of vacancies in type 316L stainless steels. J Nucl Mater 2012, 430:190-193.

134. Lu Z, Faulkner RG, Sakaguchi N, Kinoshita H, Takahashi H, Flewitt PEJ: Effect of hafnium on radiation-induced inter-granular segregation in ferritic steel. J Nucl Mater 2006, 351:155-161.

135. Kano F, Fukuya K, Hamada S, Miwa Y: Effect of carbon and nitrogen on grain boundary segregation in irradiated stainless steels. J Nucl Mater 1998, 258:1713-1717.

136. Duh TS, Kai JJ, Chen FR: Effects of grain boundary misorientation on solute segregation in thermally sensitized and proton-irradiated 304 stainless steel. J Nucl Mater 2000, 283:198-204. 137. Watanabe S, Takamatsu Y, Sakaguchi N, Takahashi H: Sink effect of grain boundary on radiation-induced segregation in austenitic stainless steel. J Nucl Mater 2000, 283:152-156. 
138. Sakaguchi N, Watanabe S, Takahashi H, Faulkner RG: A multi-scale approach to radiationinduced segregation at various grain boundaries. J Nucl Mater 2004, 329:1166-1169.

139. Kai JJ, Chen FR, Duh TS: Effects of grain boundary misorientation on radiation-induced solute segregation in proton irradiated 304 stainless steels. Mater Trans 2004, 45:40-50.

140. Sakaguchi N, Endo M, Watanabe S, Kinoshita H, Yamashita S, Kokawa H: Radiationinduced segregation and corrosion behavior on $\Sigma 3$ coincidence site lattice and random grain boundaries in proton-irradiated type-316L austenitic stainless steel. J Nucl Mater 2013, 434:6571.

141. Duh TS, Kai JJ, Chen FR, Wang LH: Numerical simulation modeling on the effects of grain boundary misorientation on radiation-induced solute segregation in 304 austenitic stainless steels. J Nucl Mater 2001, 294:267-273.

142. Field KG, Barnard LM, Parish CM, Busby JT, Morgan D, Allen TR: Dependence on grain boundary structure of radiation induced segregation in a $9 \mathrm{wt} . \% \mathrm{Cr}$ model ferritic/martensitic steel. J Nucl Mater 2013, 435:172-180.

143. Field KG, Miller BD, Chichester HJM, Sridharan K, Allen TR: Relationship between lath boundary structure and radiation induced segregation in a neutron irradiated $9 \mathrm{wt} \% \mathrm{Cr}$ model ferritic/martensitic steel. J Nucl Mater 2014, 445:143-148.

144. Marquis EA, Hu R, Rousseau T: A systematic approach for the study of radiation-induced segregation/depletion at grain boundaries in steels. J Nucl Mater 2011, 413:1-4.

145. Marquis EA: Figures 3(b) and 3(c) in Ref. [141] are incorrectly labeled: the (b) and (c) labels should be permuted. Private communication. 2015.

146. Allen TR, Tan L, Was GS, Kenik EA: Thermal and radiation-induced segregation in model Ni-base alloys. J Nucl Mater 2007, 361:174-183.

147. Marwick A, Piller R, Horton M: Radiation-induced segregation in Fe-Cr-Ni alloys. In

Dimensional Stability and Mechanical Behaviour of Irradiated Metals and Alloys. BNES

London; 1984:11-15.

148. Rothman SJ, Nowicki LJ, Murch GE: Self-diffusion in austenitic Fe-Cr-Ni alloys. J Phys FMetal Phys 1980, 10:383-398.

149. Nastar M: Segregation at grain boundaries: from equilibrium to irradiation induced steady states. Philos Mag 2005, 85:641-647.

150. Soisson F: Kinetic Monte Carlo simulations of radiation induced segregation and precipitation. J Nucl Mater 2006, 349:235-250.

151. Faulkner RG, Waite NC, Little EA, Morgan TS: Radiation-induced grain-boundary segregation in dilute alloys. Mater Sci Engr A 1993, 171:241-248.

152. Faulkner RG: Nonequilibrium grain-boundary segregation in austenitic alloys. J Mater Sci 1981, 16:373-383.

153. Nastar M, Barbe V: A self-consistent mean field theory for diffusion in alloys. Faraday Discuss 2007, 134:331-342.

154. Sastry K, Johnson DD, Goldberg DE, Bellon P: Genetic programming for multitimescale modeling. Phys Rev B 2005, 72:085438.

155. Castin N, Malerba L: Prediction of point-defect migration energy barriers in alloys using artificial intelligence for atomistic kinetic Monte Carlo applications. Nucl Instr Methods Phys Res Sec B-Beam Interactions Mater and Atoms 2009, 267:3148-3151.

156. Castin N, Malerba L: Calculation of proper energy barriers for atomistic kinetic Monte Carlo simulations on rigid lattice with chemical and strain field long-range effects using artificial neural networks. J Chem Phys 2010, 132:074507. 
157. Diaz De La Rubia T, Soneda N, Caturla MJ, Alonso EA: Defect production and annealing kinetics in elemental metals and semiconductors. J Nucl Mater 1997, 251:13-33.

158. Stoller RE, Odette GR, Wirth BD: Primary damage formation in bcc iron. J Nucl Mater 1997, 251:49-60.

159. Wirth BD, Odette GR, Maroudas D, Lucas GE: Energetics of formation and migration of self-interstitials and self-interstitial clusters in $\alpha$-iron. J Nucl Mater 1997, 244:185-194.

160. Koehler JS: Decrease in void growth-rate by interstitial trapping: J Appl Phys 1975, 46:2423-2428.

161. Nita N, Schaeublin R, Victoria M: Impact of irradiation on the microstructure of nanocrystalline materials. J Nucl Mater 2004, 329:953-957.

162. Beyerlein I, Caro A, Demkowicz M, Mara N, Misra A, Uberuaga B: Radiation damage tolerant nanomaterials. Materials Today 2013, 16:443-449.

163. Odette G, Alinger M, Wirth B: Recent developments in irradiation-resistant steels. Ann Rev Mater Research 2008, 38:471-503.

164. Demkowicz M, Bellon P, Wirth B: Atomic-scale design of radiation-tolerant nanocomposites. MRS Bulletin 2010, 35:992-998.

165. Jager W, Trinkaus H: Defect ordering in metals under irradiation: J Nucl Mater 1993, 205:394-410.

166. Ghoniem N, Walgraef D, Zinkle S: Theory and experiment of nanostructure selforganization in irradiated materials. J Comp-Aided Mater Design 2001, 8:1-38.

167. Chee S, Stumphy B, Vo N, Averback R, Bellon P: Dynamic self-organization in Cu alloys under ion irradiation. Acta Mater 2010, 58:4088-4099. 